Article

\title{
GNSS-RO Refractivity Bias Correction Under Ducting Layer Using Surface-Reflection Signal
}

\author{
Kuo-Nung Wang *, Chi O. Ao and Manuel de la Torre Juárez \\ Jet Propulsion Laboratory, California Institute of Technology, 4800 Oak Grove Drive, Pasadena, CA 91109, USA; \\ chi.o.ao@jpl.nasa.gov (C.O.A.); Manuel.Delatorrejuarez@jpl.nasa.gov (M.d.1.T.J.) \\ * Correspondence: Kuo-Nung.Wang@jpl.nasa.gov
}

Received: 13 December 2019; Accepted: 19 January 2020; Published: 22 January 2020

\begin{abstract}
Due to its high vertical resolution and cloud-penetrating capability, GNSS-Radio Occultation (RO) remote sensing technique has been utilized to observe the vertical structure of the Planetary Boundary Layer (PBL) in recent years. However, the critical refraction, or ducting, caused by large refractivity gradients usually associated with the top of the stratocumulus clouds, can negatively bias the retrieved refractivity and humidity within the PBL. Previous research has shown that combining RO retrievals and the external information, such as collocated precipitable water (PW) estimates, can effectively reduce the negative bias and enhance the retrieval quality. Nevertheless, the requirement of collocated observations from other techniques limits the applicability of this reconstruction method in practice. Here, we describe an alternative approach that uses the coherent grazing signals from the same RO event that are reflected by the Earth's surface to remove the bias due to ducting. Additional observations are no longer necessary in this approach because the reflected signals provide the extra constraint. A least squares framework is used to select the candidate from a family of solutions wherein reflected bending angle best matches the corresponding observation. This new method was validated by both multiple phase screen (MPS) simulation and the simulated RO bending angle via forward Abel transform, and it was tested with the actual GPS-RO measurements. While, in general, the reflected bending retrieved from the current mission was noisy, the results show that this approach can potentially reduce the negative bias and improve RO observation within the PBL without assistance by the external information, such as PW.
\end{abstract}

Keywords: radio occultation; GNSS; planetary boundary layer; ducting; reflection

\section{Introduction}

Global Navigation Satellite System Radio Occultation (GNSS-RO) is an atmospheric limb sounding technique to observe the vertical thermodynamic structure using GNSS signals received by low Earth orbit (LEO) satellites. When the occulted signal from the GNSS constellations propagates through the stratified atmosphere, its ray path could bend at the limb of the Earth due to the change of refractivity [1]. The bending angle of each ray path, which can be calculated by the time-varying signal phase delay observations, will be inverted to retrieve the vertical profiles of refractivity, temperature, and moisture. Since the first GNSS-RO mission, Global Positioning System/Meteorology (GPS/MET), successfully demonstrated the remote sensing technique in 1995, several new GNSS-RO instruments, including CHAMP, SAC-C, COSMIC, GRACE, and METOP, were launched and currently provide thousands of daily profiles in combination. Recently, launched GNSS-RO missions, such as COSMIC II (June 2019) [2], METOP-C (November 2018), and GRACE-FO (May 2018), were also deployed to increase the total number of $\mathrm{RO}$ atmospheric observations to more than 10000 per day and further improve the global weather prediction. 
In recent years, GNSS-RO remote sensing has been applied to planetary boundary layer (PBL) studies [3,4]. PBL, which is the bottom layer of the atmosphere $(\lesssim 2 \mathrm{~km})$, controls the energy distribution from the surface and has a great impact to both local weather and global climate through turbulent winds and cloud formation [5]. Because of its importance in weather modeling, PBL has been extensively studied for decades and is listed as one of the most targeted observables in the decadal survey [6]. GNSS-RO technique is valuable to PBL probing tasks for several reasons. First, as a spaceborne remote sensing technique, GNSS-RO can provide worldwide boundary layer information, including remote regions, with few in-situ measurements. Second, GNSS-RO provides high vertical resolution $(\sim 100 \mathrm{~m})$ [7] refractivity measurements, which are not available from other nadir remote sensing instruments [8]. Third, the L-band GNSS signals, which are designed for navigation purposes, can penetrate clouds and precipitations [9], which are common at the level of PBL height. These advantages make GNSS-RO a unique and valuable technique for PBL characterization [10-14].

However, the strong temperature inversion and moisture change at the top of PBL could induce large negative refractivity gradient and sharply increase the bending of the propagating radio signal ray path. When the bending angle is larger than the curvature of the Earth's surface, ducting occurs and the signal will be "trapped" inside the atmosphere. In general, ducting occurs when $\mathrm{dN} / \mathrm{dr} \lesssim$ -157 (N-units $/ \mathrm{km}$ ) (the region between $h_{m}$ to $h_{t}$, as shown in Figure 1), which can be frequently observed in the subtropics below $2 \mathrm{~km}$. Contrary to the ducting layer definition from von Engeln and Teixeira [15] $\left(h_{m}\right.$ to $\left.h_{t}\right)$, here, we define it as the layer between $h_{b}$ and $h_{t}$, where the radio signals within will theoretically be trapped [16]. In the case of GNSS-RO, in which both the transmitter and receiver are located outside the atmosphere, the signal path will never be "trapped" inside the ducting layer. Instead, the tangent points of $\mathrm{RO}$ ray paths skip the ducting altitudes and cause the bending angle information loss inside the ducting layer. As a result, the refractivity retrieval process becomes an ill-posed inversion problem (i.e., multi-refractivity solutions correspond to the same bending angle profile) when ducting is present. The refractivity profile retrieved by standard Abel inversion, which assumes no ducting occurs and no bending angle gap within the ducting layer, will be negatively biased ( $N$-bias) below the ducting $\left(h_{t}\right)$, as shown in Figure 1 [16-18]. Note that only the elevated ducts $\left(h_{b}>0\right)$ condition could cause the $N$-bias, whereas in the surface and evaporation duct cases, the RO profile would be cut-off at $h_{t}$, resulting in higher penetration altitude. Ao [16] and Xie et al. [18] stated that the N-bias caused by the ducting can be as much as $5 \%$ in several subtropical ocean basins. Therefore, it is essential to remove the $\mathrm{N}$-bias in the retrieval profiles due to ducting for correctly characterizing PBL using GNSS-RO.

It is worth clarifying here that ducting is not the only cause of the GNSS-RO N-bias. Tracking error [19], cycle slips [20], unbalanced noise spectrum [21], and atmospheric turbulence [22] could also lead to both bending angle and $\mathrm{N}$-bias in the lower-altitude RO retrievals. It is difficult to identify and distinguish different sources of $\mathrm{N}$-bias solely by $\mathrm{RO}$ observations, and in this study, we only focus on correcting the $\mathrm{N}$-bias caused by ducting. Several efforts were made to reduce the negative bias. Xie et al. [18] analytically proved that, when ducting occurs, one bending angle profile w.r.t. impact parameter can correspond to an infinite number of refractivity profile solutions. These refractivity solutions can be described by a single non-linear function which depends on the thermodynamic structure within the ducting layer. While the structure inside the ducting layer cannot be directly known from the GNSS-RO signal observations, one can use physical constraints to identify the correct profile from the solution continuum. The approach proposed by Xie et al. [18] constrains the solution by assuming GNSS-RO profiles can reach all the way down to the Earth's surface and cut-off right at the surface level. In practice, however, the lowest point of GNSS-RO bending angle profiles do not often reach the surface due to measurement noise and tracking error [10], which makes the application of this constraint difficult, in practice. As an alternative constraint, Wang et al. [23] proposed distinguishing different profiles in the continuum with the integrated precipitable water (PW) and using the collocated external PW retrievals from other instruments as the constraint. This approach requires collocated 
observations, which are not always available and could induce the collocation error in the $N$-bias correction process.

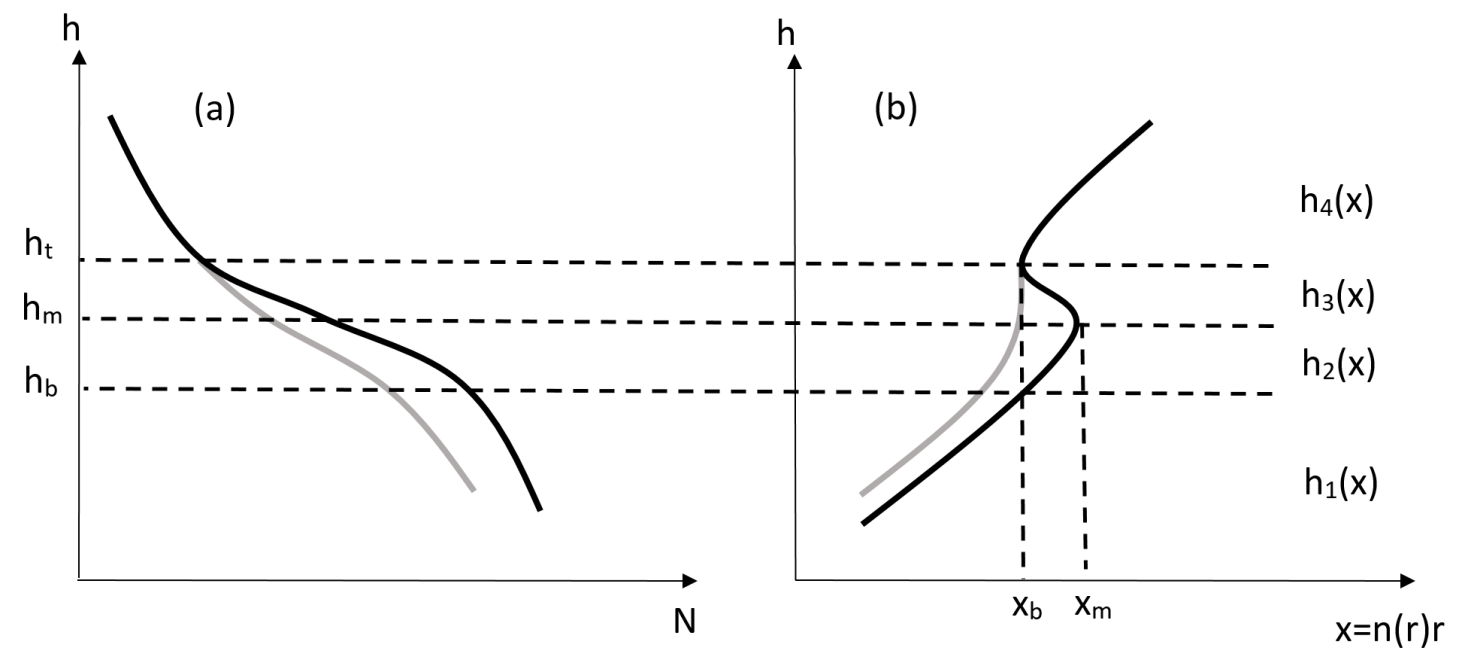

Figure 1. The illustration of the corresponding refractivity profile w.r.t height (a) and the height function w.r.t $x=n(r) r(\mathbf{b})$ when ducting occurs. The true profiles are shown in black, and the profiles acquired by Global Navigation Satellite System Radio Occultation (GNSS-RO) Abel retrievals are shown in gray. The large refractivity gradient inside the ducting layer, located between $h_{b}$ and $h_{t}$, causes the negative slope of $h_{3}(x)$ and the multi-valued function $h(x)$ between $x_{b}$ and $x_{m} . h_{m}$ is the height where the local maximum $x_{m}$ occurs. $h(x)$ can be divided into four different curves $\left(h_{1}(x)\right.$ to $\left.h_{4}(x)\right)$ by $h_{b}, h_{m}$, and $h_{t}$.

In this paper, we introduce the concept of using reflected GNSS-RO signal for N-bias removal. Reflection component of the GNSS-RO signal can be easily detected (more than $45 \%$ of the cases) in the ocean basins with latitude greater than 20 degrees [24]. While the bending angle of the direct signal for each $N$ profile in the continuum is the same, the corresponding reflected bending angle is distinctive and can be used to discriminate different profiles when ducting occurs. This concept will be further explored in Section 2 with Multiple Phase Screen (MPS) simulation, along with the reflected bending angle calculation. Since the reflection component can be extracted directly from the standard RO observations, no external information is required to set up a constraint. In Section 3, the least square approach using reflected bending angle as the constraint for choosing the unbiased refractivity solution is illustrated. The retrieval results in end-to-end simulation and actual cases will be shown in Section 4. Some restrictions of applying reflected bending as the constraint are discussed in Section 5. The conclusion is provided in Section 6. All the variables used in the main text are listed and defined in Table 1.

Table 1. Symbols used throughout the main text of the article.

\begin{tabular}{cccc}
\hline Symbol & Definition & Symbol & Definition \\
\hline$\alpha_{D}$ & Direct bending & $\alpha_{R}$ & Reflected bending \\
$\alpha_{R}^{C}$ & reflected bending candidate & $\alpha_{R}^{O}$ & Observed reflected bending \\
$\Phi_{D}$ & Direct signal phase & $\Phi_{R}$ & Reflected signal phase \\
$\phi$ & grazing angle & $\rho_{s}$ & Reduction factor \\
$a$ & Impact parameter & $a_{S}$ & Impact parameter at the surface \\
$A_{D}$ & Direct signal amplitude & $A_{R}$ & Reflected signal amplitude \\
$d_{t h}$ & Ducting layer thickness & $g$ & Rayleigh roughness parameter \\
$h$ & Height & $h_{b}$ & Height of ducting layer (bottom) \\
$h_{m}$ & Height of ducting layer (middle) & $h_{t}$ & Height of ducting layer (top) \\
\hline
\end{tabular}


Table 1. Cont.

\begin{tabular}{cccc}
\hline Symbol & Definition & Symbol & Definition \\
\hline$h_{\text {cos }}$ & Cosine window width & $i$ & Level index number \\
$N$ & True refractivity & $N_{A I}$ & Abel-inverse refractivity \\
$N_{R A O B}$ & Radiosonde refractivity obs. & $S_{h}$ & Surface standard deviation \\
$n$ & True refractive index & $n_{A I}$ & Abel-inverse refractive index \\
$r$ & Radius & $r_{E}$ & Earth's radius \\
$s$ & Total signal & $s^{\prime}$ & Frequency shifted signal \\
$t$ & Time & $w$ & Window function \\
$x$ & $n(r) r$ & $x_{b}$ & $x$ value at bottom of ducting layer \\
$x_{m}$ & Local maximum of $x$ & $\lambda$ & Signal wavelength \\
\hline
\end{tabular}

\section{Reflecting Signal and Ducting}

To illustrate that refractivity profiles are under-determined from the bending angle measurements in the presence of ducting, a simple example using Multiple Phase Screen (MPS) simulation is shown in Figure 2. A ducting layer located at $2 \mathrm{~km}$ can be simulated using a nonlinear function [25], as the blue line is shown in Figure 2a:

$$
N(h)=350 \exp \left(\frac{-h}{7}\right) \times\left[1-\frac{0.2}{\pi} \arctan \left[\frac{h-2}{0.06}\right]\right],
$$

where $N$ is the refractivity (N-unit) as a function of the height $h(\mathrm{~km})$. The minimum gradient $d N / d h$ can reach $-250 N$-units $/ \mathrm{km}$, which is less than the critical ducting value $-157 \mathrm{~N}$-units $/ \mathrm{km}$. The corresponding bending angle $\alpha$, with respect to the impact parameter $a$ from the direct signals, can be calculated using a generalized forward Abel transform $[17,18]$ to integrate the multi-value function $n(a)$ :

$$
\alpha_{D}(a)=-2 a\left[\int_{a}^{x_{m}}+\int_{x_{m}}^{x_{b}}+\int_{x_{b}}^{\infty}\right] \frac{1}{n(x)} \frac{d n(x)}{d x} \frac{d x}{\sqrt{x^{2}-a^{2}}},
$$

where $x_{m}$ is the local maximum of $x$, and $x_{b}$ is the critical value when ducting starts occurring. The subscript $D$ is added in Equation (2) to identify it as the bending angle of the direct ray path. The forward transformed bending angle profile, shown in Figure $2 b$, simulates the RO bending angle observation derived from direct GNSS signal paths. Note that the bending angle increases sharply at the height where the ducting occurs. One can apply the standard Abel-inversion operation to the simulated bending angle:

$$
\begin{gathered}
n_{A I}(x)=\exp \left[\frac{1}{\pi} \int_{x}^{\infty} \frac{\alpha_{D}(a) d a}{\sqrt{a^{2}-x^{2}}}\right], \\
N_{A I}(h)=\left[n_{A I}(h)-1\right] \times 10^{6},
\end{gathered}
$$

where $n_{A I}$ is the Abel-inverse refractivity. Since the Abel-inversion assumes the variable $x(r)=n r$ is a single-valued function and the multi-valued portion in the profile is ignored, the resulting refractivity $N_{A I}(h)$ could contain a large negative bias compared to the true profile $N(h)$, as shown in Figure 2a. The fact that both refractivity profiles correspond to the same bending angle indicates that using only the direct GNSS RO signal cannot distinguish them in the retrieval process when ducting occurs. Unlike the approach that relies on the external information provided by other techniques to distinguish an individual profile, the feasibility of using a GNSS-RO signal itself that reflected from the the Earth's surface was assessed in this research. 


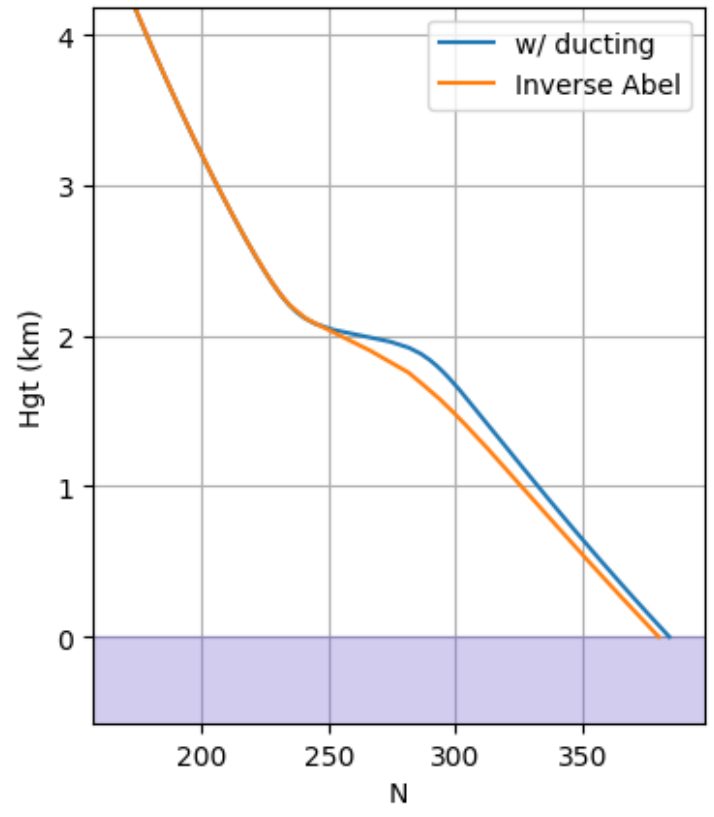

(a)

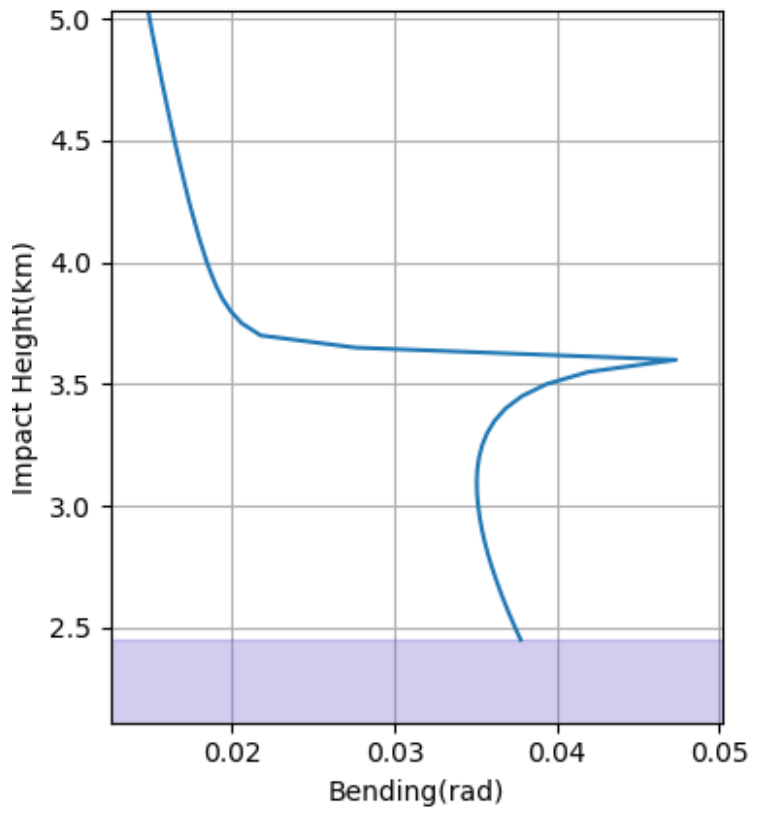

(b)

Figure 2. (a) The refractivity profile with duct (blue color) is used to simulate the RO signal phase by multiple phase screen (MPS) . The retrieved profile of the MPS signal by the Abel-inversion (orange color). The strong $N$-bias is shown below the $2 \mathrm{~km}(\mathbf{b})$ bending angle profile, corresponding to both cases-when ducting occurs, the direct bending angle is indistinguishable.

Here, we simulate the reflected GNSS-RO signals by following the steps of Beyerle et al. [26] to perform the MPS simulation with the refractivity profiles shown above. MPS modeled the atmospheric refractivity as a series of screens (as shown in Figure 3), and the propagating GNSS signal amplitude and phase through the screens can be calculated by iterating Fourier Transform and inverse Fourier Transform at each screen [25]. In our approach, there are $2^{19}$ grid points for each screen that expands from $-274.288 \mathrm{~km}$ to $250 \mathrm{~km}$ vertically, as well as 4001 phase screens in total from $-1000 \mathrm{~km}$ to $3000 \mathrm{~km}$ horizontally. The interval between each grid is $1 \mathrm{~m}$, and the horizontal distance between each screen is $1 \mathrm{~km}$. The radius of the Earth is set as $6370 \mathrm{~km}$. In the last (observation) screen, we assume the vertical speed of the satellite is $2.7 \mathrm{~km} / \mathrm{s}$ corresponding to the satellite with orbit altitude $\sim 450 \mathrm{~km} \mathrm{[26].}$

The resulting complex signal $s(t)$, which is the combination of direct and reflected signals, can be written as a function of time $t$ :

$$
s(t)=A_{D}(t) \exp \left[i \Phi_{D}(t)\right]+A_{R}(t) \exp \left[i \Phi_{R}(t)\right],
$$

where $A_{D}$ and $A_{R}$ are the direct and reflected sub-signal amplitude, and $\Phi_{D}$ and $\Phi_{R}$ are the direct and reflected sub-signal phase, respectively. By adding a cosine window function above the Earth's surface for each screen in the MPS simulation:

$$
w(h)= \begin{cases}1 & : h>h_{\cos } . \\ \cos ^{2}\left(\frac{\pi}{2} \frac{h_{c o s}-h}{h_{\cos }}\right) & : 0<h<h_{\cos } . \\ 0 & : h<0 .\end{cases}
$$

one can remove the reflected signal component (second term in Equation (5)) from $s(t)$ and extract $\Phi_{D}(t)$. The variable $h_{c o s}$, which is the layer width where the cosine window applies, is set as $2 \mathrm{~km}$ in 
this research. With this direct signal phase information, the spectrum of the signal $s(t)$ can be shifted by the following equation and centered on the direct frequency, which is shown in Figure 4 .

$$
s^{\prime}(t)=\frac{s(t)}{\exp \left[i \Phi_{D}(t)\right]}=A_{D}(t)+A_{R}(t) \exp \left[i\left(\Phi_{R}(t)-\Phi_{D}(t)\right)\right] .
$$

As shown in Figure 4a, the signal consists of several frequency components due to different ray paths in the presence of ducting. The component located at zero-frequency goes through the direct ray path (Path 1 in Figure $4 \mathrm{~b}$ ), and the lower amplitude component shown before $85 \mathrm{~s}$ is the reflected sub-signal (Path 4 in Figure 4b). Because of the $50 \mathrm{~Hz}$ sampling rate, the reflected sub-signal is aliased before $50 \mathrm{~s}$ and gradually converges with the direct signal from 50 to $85 \mathrm{~s}$, as a result of the closer ray path length between direct and reflected signals in the low elevation geometry. The direct signal vanishes at about $80 \mathrm{~s}$ because of strong defocusing at the ducting layer, but the signal component from the path below the ducting layer (Path 2 and 3 in Figure $4 \mathrm{~b})$ takes over in a relatively short time $(\sim 82 \mathrm{~s})$. This component, which starts at the frequency of $\sim-2 \mathrm{~Hz}$, breaks into two different branches due to the strong refractivity gradient of ducting. One is closer to the ducting layer (Path 2 in Figure $4 \mathrm{~b}$ ) and dominates the received RO signal (located at zero-frequency) from $85 \mathrm{~s}$ to the end of the occultation period. The other one approaches to the Earth's surface (Path 3 in Figure $4 \mathrm{~b}$ ) and eventually combines with the reflected component at around $85 \mathrm{~s}$, when the tangent points of both sub-signal ray paths reach the surface and stop tracking. It should be emphasized that the tangent points of the reflected signals are always located below the surface, which make the reflected signals an effective constraint since no information gap will be formed due to ducting.

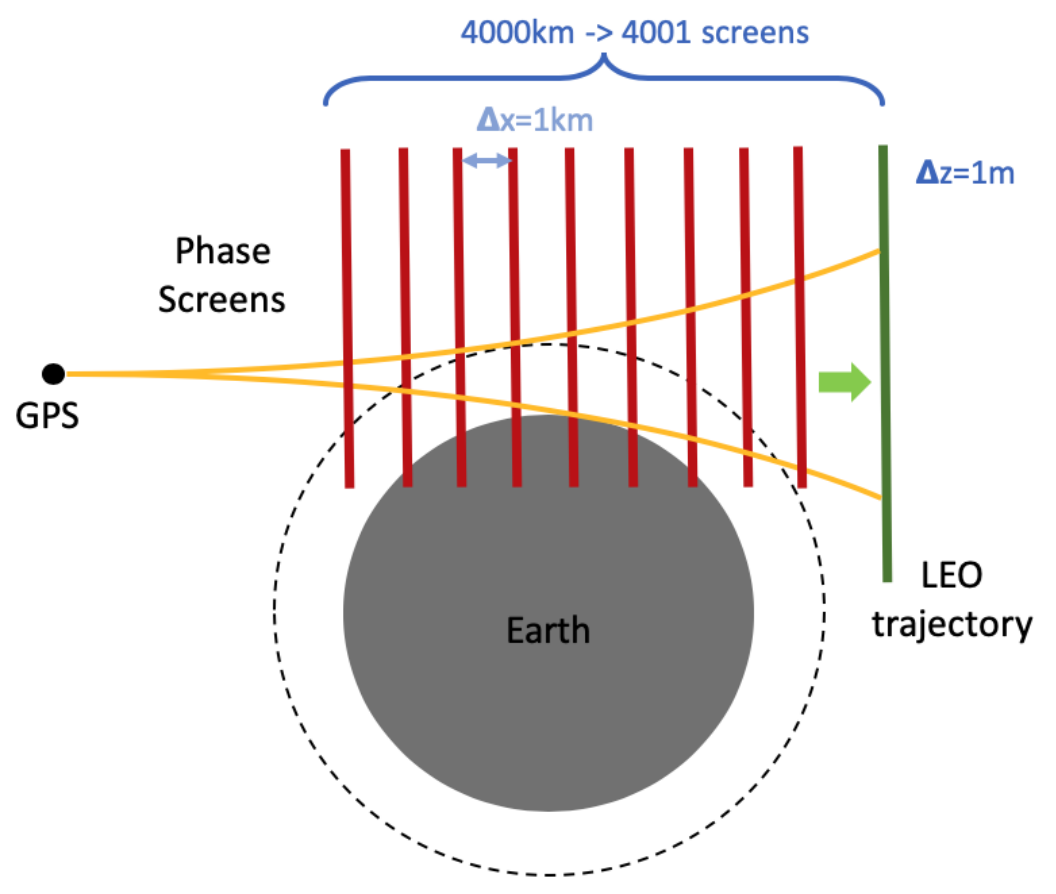

Figure 3. Illustration of the MPS simulation. Four thousand phase screens (red color) are used to simulate the propagating GNSS signals through the atmosphere and arrives at low Earth orbit (LEO) trajectory (green color). The details of the simulation are provided in texts. 


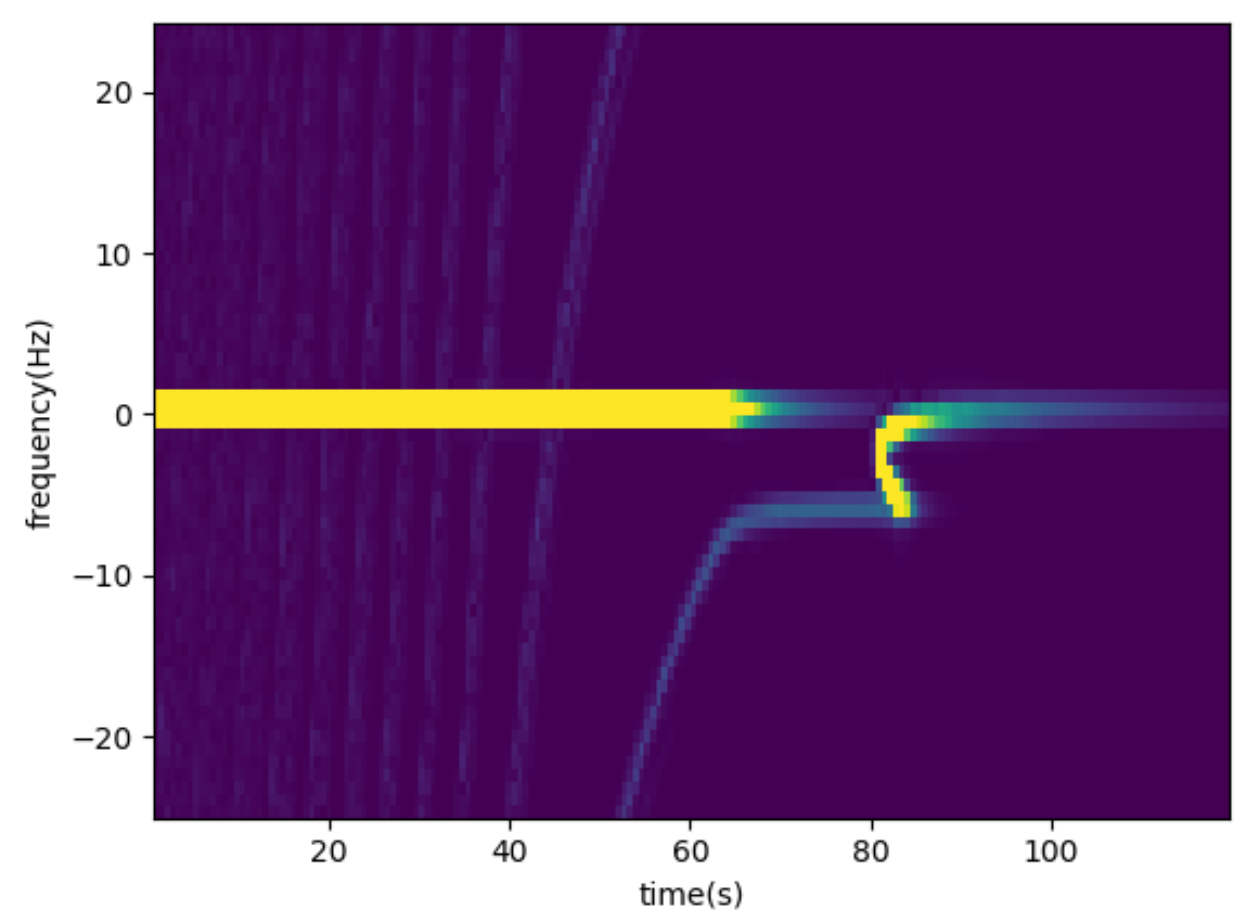

(a)

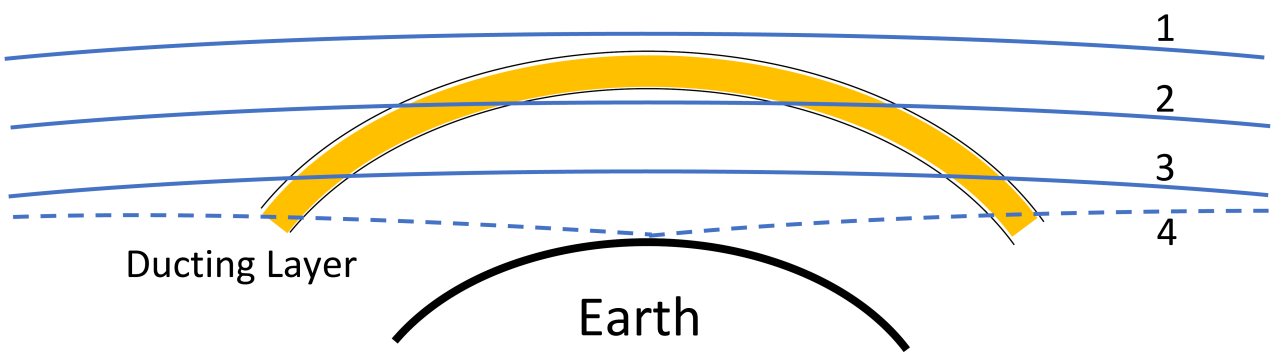

(b)

Figure 4. (a) The spectrum of the signal when ducting occurs. Strong signal at zero frequency comes from the direct $\mathrm{RO}$ ray path. The reflected signal shows up as the low strength component in the negative frequency band from 50 to $80 \mathrm{~s}$. The weak signal component before $50 \mathrm{~s}$ is the aliased reflected signal. The strong component around $80 \mathrm{~s}$ is the direct signal below the ducting layer. (b) The corresponding geometry of the direct and reflected ray paths in ducting condition.

In this paper, the reflected GNSS-RO signals are utilized by calculating their bending angles. The bending angle of a reflected $\mathrm{RO}$ signal path (subscript $\mathrm{R}$ ) based on a known ducted refractivity profile can be defined as [24]:

$$
\alpha_{R}(a)=-2 a\left[\int_{a_{S}}^{x_{m}}+\int_{x_{m}}^{x_{b}}+\int_{x_{b}}^{\infty}\right] \frac{1}{n(x)} \frac{d n(x)}{d x} \frac{d x}{\sqrt{x^{2}-a^{2}}}-2 \arccos \left(\frac{a}{a_{S}}\right),
$$

where $a_{S}$ is the impact parameter at the surface. The first term of Equation (8) describes the ray path bending due to the ducted atmosphere above the surface, while the second term is caused by the reflection of the surface. Note that the integration interval starts from the surface instead of the regular ray path impact parameter, which in the case of reflection, is located below the surface. Here, we focus on the reflections from a horizontal surface (e.g., ocean surface) without tilting so that the reflection points shifting described in Beyerle et al. [26] is not considered in this analysis. The roughness of the 
sea surface has limited impact in this case since the grazing angle is relatively small $\left(\sim 1^{\circ}\right.$ when the impact height is $1 \mathrm{~km}$ below the surface).

The bending angle of each reflected path can be directly calculated from the received signal amplitude and phase via the current radio-holographic method, like phase matching (PM), by extending the impact parameter range below the surface [27]. Applying PM to the MPS, simulated signals result in both direct and reflected bending angles for the corresponding refractivity profile. In this analysis, the two refractivity profiles described by Equation (1), (4) are used in separate MPS simulations, and both direct and reflected bending angle for each refractivity profile are calculated by PM. The results are shown in Figure 5. The portion of the bending angle where the impact parameter range is above the surface (non-shaded) is contributed by the direct GNSS-RO signals, while the part below the surface (shaded) comes from the reflected GNSS-RO signal components only. Two bending angle profiles corresponding to the refractivity profile of Equation (1) (blue line) and Equation (4) (orange line) align to each other above the surface, which is consistent with our former analysis, shown in Figure 2. However, a large difference between these two reflected bending angle profiles can be found below the surface. The difference can reach $0.005 \mathrm{rad}$ in reflected bending or $0.3 \mathrm{~km}$ in the impact parameter close to the surface and gradually decreases below. This difference in the reflected bending angle profile can thus be used to distinguish different profiles corresponding to the same direct bending angle in the ducting situations. Note that this is a useful feature for weather forecast data assimilation, which can identify or flag a specific case if it is contaminated by the ducting-caused $\mathrm{N}$-bias [28]. Here, we take this idea further to reconstruct the refractivity profile and reduce the $\mathrm{N}$-bias, and the algorithm to identify the correct profile with the reflected bending angle is introduced in the next section.

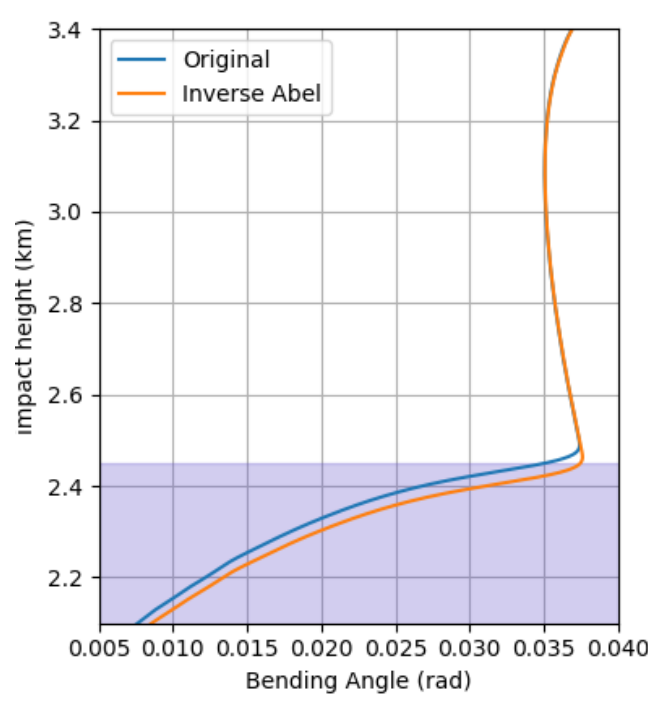

(a)

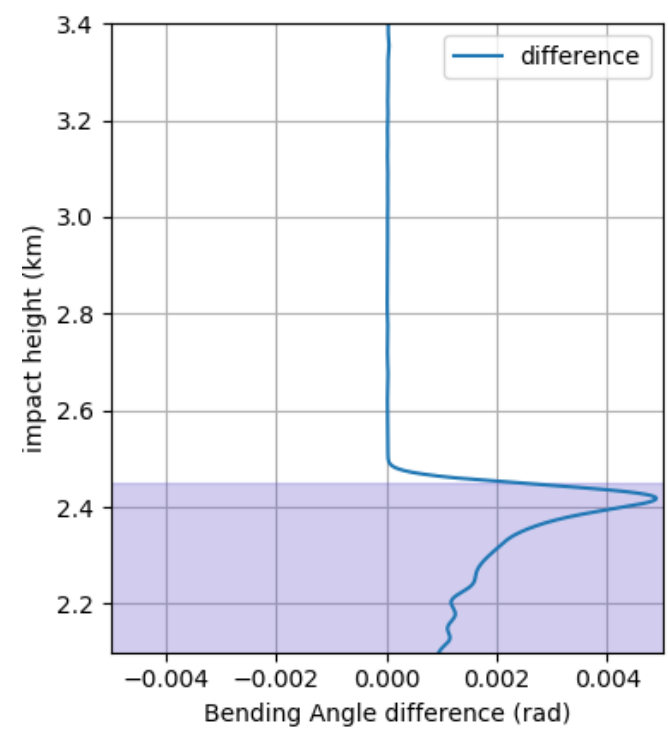

(b)

Figure 5. (a) Bending angle profiles of the corresponding refractivity, with the same color code in Figure 2. (b) The difference between the two bending angle profiles. The impact parameter in the shaded area is below the surface, where the reflected bending angle is located. As described in Section 2, when ducting occurs, the bending angle of the direct signal has zero difference, while the reflected bending angle profiles are distinguishable.

\section{Bias Correction}

\subsection{Family Solution and Reflected Bending Angle}

Xie et al. [18] proposed that, when ducting occurs, an infinite number of refractivity profiles can correspond to a single bending angle observation which caused an ill-posed inversion problem. 
Given an Abel-inverse refractivity profile, one can analytically derive the "family" of the candidate profiles $N_{f}(h, d x)[16,18]$, as shown in Appendix A, where $d x=x_{m}-x_{b}$. While, in Wang et al. [23], two independent variables were used to account for the uncertainty of $x_{b}$, here, we assume the $x_{b}$ a priori is accurate enough and only one independent variable $x_{m}$ is necessary to simplify the process. Since the measurement error of $x_{b}$ is usually small and unbiased relative to the a priori profiles [23], the results are not sensitive to this simplification. The family of refractivity profiles derived from the Abel-inversion results of Equation (4), with respect to different $x_{m}$, is shown in Figure 6a. As shown in the figure, the black, dotted curve retrieved by standard Abel-inversion is negatively biased at the ducting altitude compared to the refractivity profile depicted by Equation (1), which is shown as the blue, solid line. The dashed lines in different colors are the family of the refractivity solutions $N_{f}(h, d x)$ with different $d x$ values based on the Abel inversion result in Equation (4) and are given ducting height $(2 \mathrm{~km})$. As described above, the Abel-inversion process assumes no ducting occurs; therefore, its profile corresponds to the case with $d x=0$. Since all the candidates (including the truth and the Abel-inverse solutions) correspond to the same direct bending angle profile, it is impossible to identify the correct solution in the family solely using direct bending angle observation.

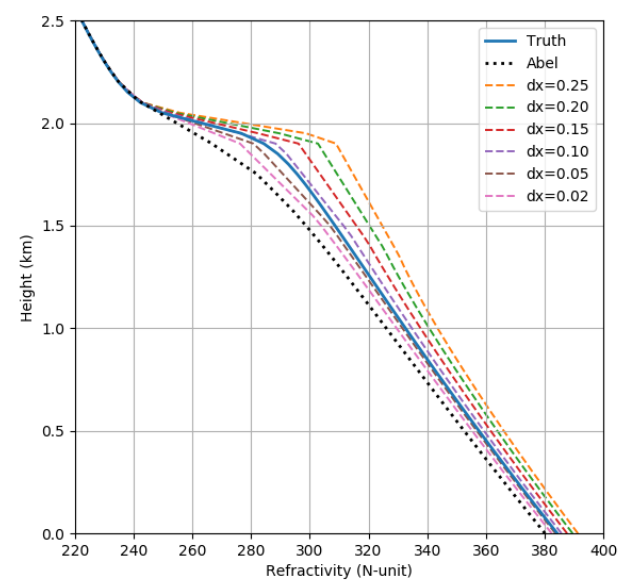

(a)

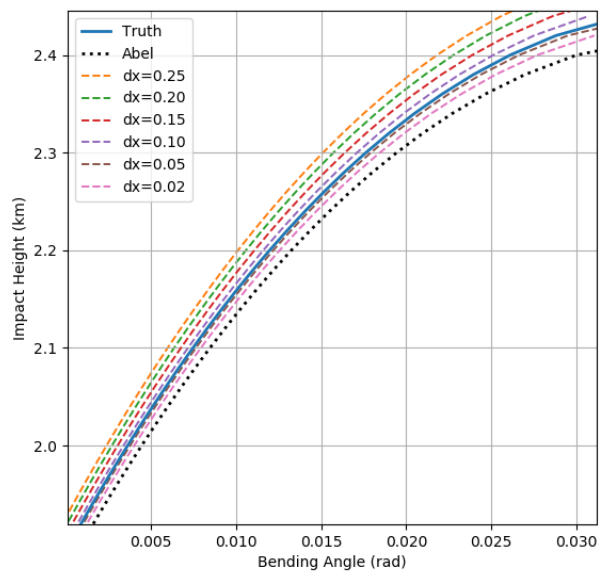

(b)

Figure 6. The profiles in the same family: (a) refractivity profile and (b) reflected BA profile, shown in dashed lines with different $d x$. The true profile are shown as solid blue line. The Abel-inverse profile is shown as the black dotted line which contains the largest negative bias in the family

To distinguish each individual profile in the family, the reflected bending angle of each refractivity candidate $N_{f}$ can be calculated by the forward model of Equation (8). However, two issues have to be addressed in practice. First, $\mathrm{RO}$ profiles can be cut-off before the tangent point reaches the surface due to low signal-to-noise ratio (SNR). The RO refractivity profiles do not start from the surface in most of the cases, consequently making the first integral in Equation (8) incomplete. Here, we extrapolate each $N_{f}$ by fitting the lowest $0.5 \mathrm{~km}$ exponentially to extend it to the surface before calculating its reflected bending angle. This is reasonable because it is frequently observed that the moisture within the boundary layer is well-mixed. A simple error analysis regarding the extrapolation is given in Appendix B. Second, the computational cost of the reflected bending forward model at all levels is relatively high. Since the optimization problem of choosing the best candidate profile should be solved iteratively (which will be stated in the next subsection), a more efficient forward model implementation is required. In this research, the approximate approach proposed by Healy and Thepaut [29], which assumes the refractivity profile $N(x)$ varies exponentially at each interval, is used:

$$
\Delta \alpha_{R, i}(a)=10^{-6} \sqrt{2 \pi a p_{i}} N_{i} \exp \left\{p_{i}\left(x_{i}-a\right)\right\}\left[\operatorname{erf}\left\{\sqrt{p_{i}\left(x_{i+1}-a\right)}\right\}-\operatorname{erf}\left\{\sqrt{p_{i}\left(x_{i}-a\right)}\right\}\right],
$$


where $i$ is the level index number, $\operatorname{er} f$ is the error function, and $p_{i}$ is defined as:

$$
p_{i}=\frac{\ln \left(N_{i} / N_{i+1}\right)}{x_{i+1}-x_{i}}
$$

Note that, in the reflected bending angle calculation, the impact parameter $a$ is always below the surface, and unlike the case of the direct bending angle, all levels above the surface are summed. At the height of ducting layer, the variable $p_{i}$ can fluctuate significantly due to a large gradient of refractivity and small change of the $x$, which cause the assumption of exponential refractivity curve to be invalid. In this case, when $p_{i}<0$ (increasing $N$ or decreasing $x$ w.r.t. height $h$, the latter could occur when $N(h)$ has a sharp decreasing slope during ducting) or $p_{i}>2$ (only slight change of $x$ w.r.t. height $h$ at the local maximum of $x(h)$ when ducting occurs), the linear shape of $N(x)$ is assumed instead, and Equation (9) should be modified as:

$$
\Delta \alpha_{R, i}(a)=-2 \sqrt{2 a} 10^{-6} \frac{N_{i+1}-N_{i}}{x_{i+1}-x_{i}}\left(\sqrt{x_{i+1}-a}-\sqrt{x_{i}-a}\right),
$$

and the total reflected bending with given impact parameter $a$ below the surface can be calculated as:

$$
\alpha_{R}(a)=\sum_{i} \Delta \alpha_{R, i}(a)
$$

The resulting reflected bending angle of each refractivity profile in the family of Figure $6 \mathrm{a}$ is shown in Figure 6b. It is shown in the figure that the reflected bending angle profiles are not the same for each corresponding refractivity profile in the family. Actually, larger refractivity profiles in the family have smaller reflected bending angle profiles at the same level or a larger impact parameter, and vice versa. This characteristic makes the reflected RO signal a suitable constraint to distinguish different profiles below the ducting layer.

Based on Figure 6, the true profile lies between $d x=0.05$ to $d x=0.10$. To search for the exact $d x$ value and its corresponding profile solution, the least square approach is used, as stated in the following subsection.

\subsection{Least Squares Estimation}

Unlike the approach proposed by Wang et al. [23], which used optimal estimation to estimate both $d x$ and $x_{b}$ simultaneously, here, $d x$ is the only variable to be estimated which does not have an a priori; hence, a simple non-linear least squares can fit the needs here. The reflected bending angle of the candidate profile $\alpha_{R}^{C}$ for each iteration is calculated by the forward model described in Section 3.1. The trust region reflective algorithm is used in this research for non-linear least squares implementation to search for the $d x$ that could give the minimum reflected bending gradient difference between the candidate and the observation:

$$
d x=\operatorname{argmin} \sum_{a}\left[\frac{d \alpha_{R}^{C}(a)}{d a}-\frac{d \alpha_{R}^{O}(a)}{d a}\right]^{2},
$$

where argmin means the argument of the minimum. $\alpha_{R}^{O}$ is the reflected bending angle observation calculated by PM or other bending angle retrieval methods from the excess phase measurements. The reason that the bending gradient is chosen over the bending angle as the cost function is that, in reality, the refractivity profile cannot be well-represented by the simple bilinear model, and the calculated reflected bending angle, which mostly contributed by ducting when tangent height was 
located right below the surface, is sensitive to this difference. On the other hand, the bending gradient can be written as [30]:

$$
\frac{d \alpha_{R}(a)}{d a} \approx-\sqrt{\frac{1}{2 r_{E}}} \frac{d_{t h}}{h_{m}^{3 / 2}}+\sqrt{\frac{2}{r_{E}\left(a_{S}-a\right)}},
$$

where $d_{t h}$ is the thickness of the ducting layer. $a_{S}$ is the impact parameter at the surface, and $r_{E}$ is the radius of the Earth. The first term described the bending gradient due to atmospheric refraction and is dominated by the ducting structures. But the main contribution of the bending gradient comes from the reflection described by the second term, when tangent heights are located close to (and below) the surface. Therefore, the bending gradient will be insensitive to the difference in ducting structure between model and observation, as well as better-related to the refractivity at surface that is determined by $x_{m}$. An example using two different criteria will be provided in the next section.

By defining the cost function as Equation (13), the profile with the corresponding reflected bending angle that best matches the slope of observation will be selected. In practice, the reflected RO signal will be significantly attenuated when its frequency deviates from the direct signal because the PRN code no longer aligns with the one locally generated in the open-loop receiver. In a setting case, this happens in the beginning stage of the occultation, which causes the absence of the reflected bending when $a<a_{s}-0.5$. Additionally, the interference by the direct signal around the surface could spread the spectrum at the impact parameter $a_{s}$. Therefore, we only compute the bending difference between $a_{s}-0.4 \mathrm{~km}$ to $a_{s}-0.1 \mathrm{~km}$ and use it as the observation for least squares.

Figure 7 shows the reconstructed result of the same case stated in the previous section using the least squares approach. The reflected bending angle profiles $\alpha_{R}^{O}$ (True) and the best candidate $\alpha_{R}^{C}$ selected by least squares (Fwd) are shown in Figure 7a. The least square chose the $d x$ that leads to the minimum difference of the two curves between the range of $a_{s}-0.4$ and $a_{s}-0.1$, which are indicated as black, dashed lines. The resulting refractivity profile is shown in Figure $7 \mathrm{~b}, \mathrm{c}$. As indicated in the figures, the profiles chosen by least squares are able to reconstruct the multi-valued function $x(h)$, which was erroneously taken as a single-valued function in Abel inversion. It is shown that the least squares can successfully determine the correct profile in the family and reduce the $N$-bias due to ducting.

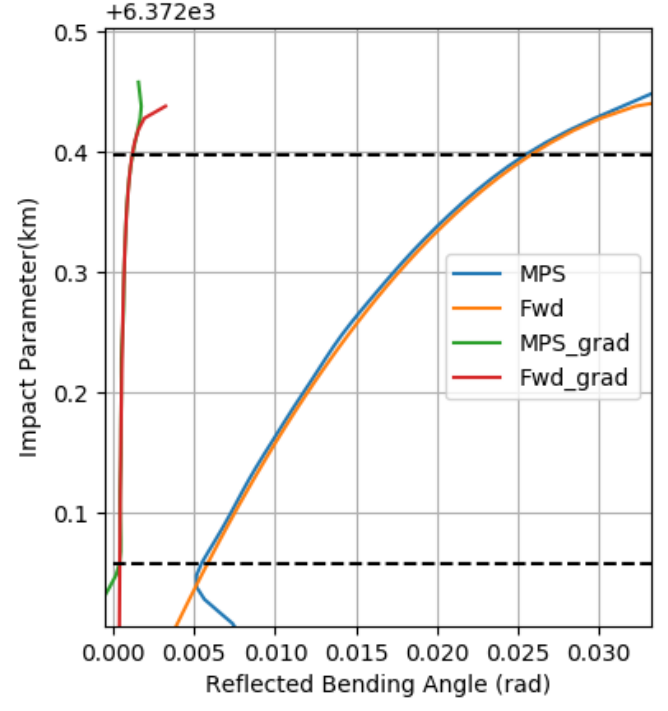

(a)

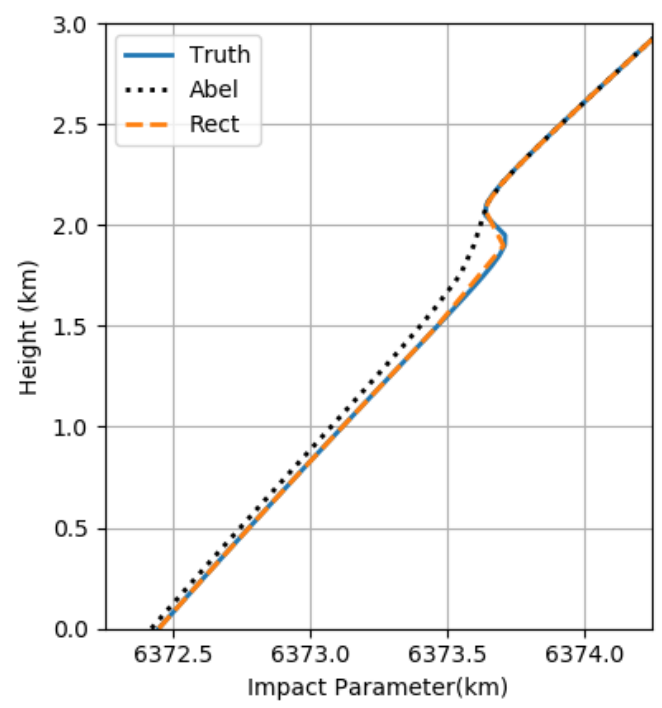

(b)

Figure 7. Cont. 


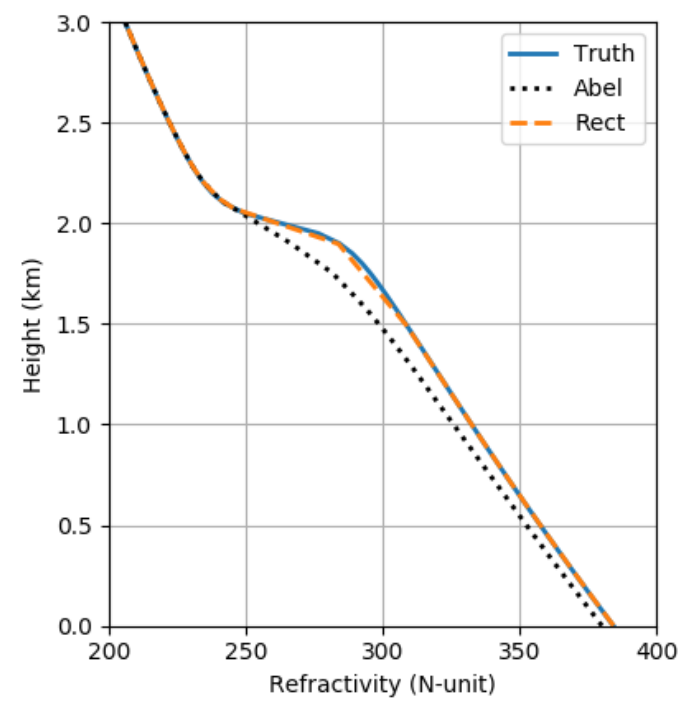

(c)

Figure 7. The reconstruction result using Least Squares approach: (a) reflected bending angle, simulated by MPS (blue color) and selected form the family (orange color). The gradient of each is shown in green and red color (b); $x$-h curve of the true profile (blue color), Abel-inverse profile (black color, dotted) and the reconstructed profile (orange color, dashed). The Abel-inverse profile remains monotonic over impact parameter, which caused the negative bias below. The reconstructed profile successfully reduces the $N$-bias. (c) Resulting refractivity profile with the corresponding (b).

\section{Results}

\subsection{End-to-End Simulation}

To verify the reconstruction algorithm described in Section 3, we conducted the same end-to-end experiment as stated in Wang et al. [23] and Xie et al. [18]. The refractivity data set from the radiosonde measurements of Variability of the American Monsoon Systems (VAMOS) Ocean-Cloud-Atmosphere-Land Study (VOCALS) campaign [31], which is located at one of the regions that has the highest frequencies of ducting conditions [32] and leads to a large N-bias in RO refractivity retrievals [33], was used to simulate both direct and reflected bending angles. As shown by Wang et al. [23], 20 cases with strong refractivity gradient at the PBL top have been selected for statistical analysis, and six among them are shown in Figure 8. The blue lines in Figure 8 are refractivity profiles from the radiosonde observations (RAOB) data, which are taken as the truth. It can be observed in these cases that the refractivity profiles has a sharp gradient at the height about $1.5 \mathrm{~km}$. By applying the forward Abel to generate the direct bending angle and simulate the standard RO refractivity observation (black, dashed line) by Abel inversion, the divergence beneath the top of PBL to the surface can be clearly observed. The corresponding direct bending angle of the true profile (RAOB) and the reflected bending angle of both true and Abel inversion profiles calculated by forward Abel are shown in Figure 9.

The reconstructed refractivity for each case is then estimated by the least square approach stated above, based on the negatively biased Abel inverse profiles. This approach looks for the profile candidate with the proxy of $x_{m}$, in which the reflected bending angle (shown as orange, solid lines in Figure 9) derivative in the shaded area best matches the simulated true reflected bending angle from the RAOB observations (blue, dotted line in Figure 9). The reconstructed refractivity results are shown as orange, solid lines in Figure 8. As the results suggested, the reconstruction can successfully correct the $\mathrm{N}$-bias below the ducting layer compared to the Abel-inverse profile by $5 \%$. Note that the reconstructed profile inside the ducting layer comes from modeling, instead of the data itself, due 
to the information gap mentioned in the Introduction. Because the assumption of bilinear structure and the constraint of calculating $x_{m}$ and $h_{b}$ stated in Appendix A cannot absolutely represent the true thermodynamic structure inside the ducting layer, the resulting profile around the top of PBL (1.5 km) does not align with the RAOB observations. Since the ducting layer only contributes a fraction of the reflected bending angle derivative compared to the surface reflection [30], this discrepancy does not much affect the estimation based on the bending angle derivative constraint, and the reconstructed profile below ducting can still remove most $N$-bias. However, this discrepancy could affect the result if the bending angle difference, rather than bending angle derivative difference, is used as the cost function. The reconstruction algorithm could erroneously choose the "best-matched" profile, but with a different surface impact parameter, and result in poor estimation. An example of using bending angle difference is provided in Figure 10. It can be observed that the chosen bending angle profile intersects with the simulated observation, which indicates that the gradient is relatively different. The estimation result is thus severely biased down to the surface compared to the true profile.
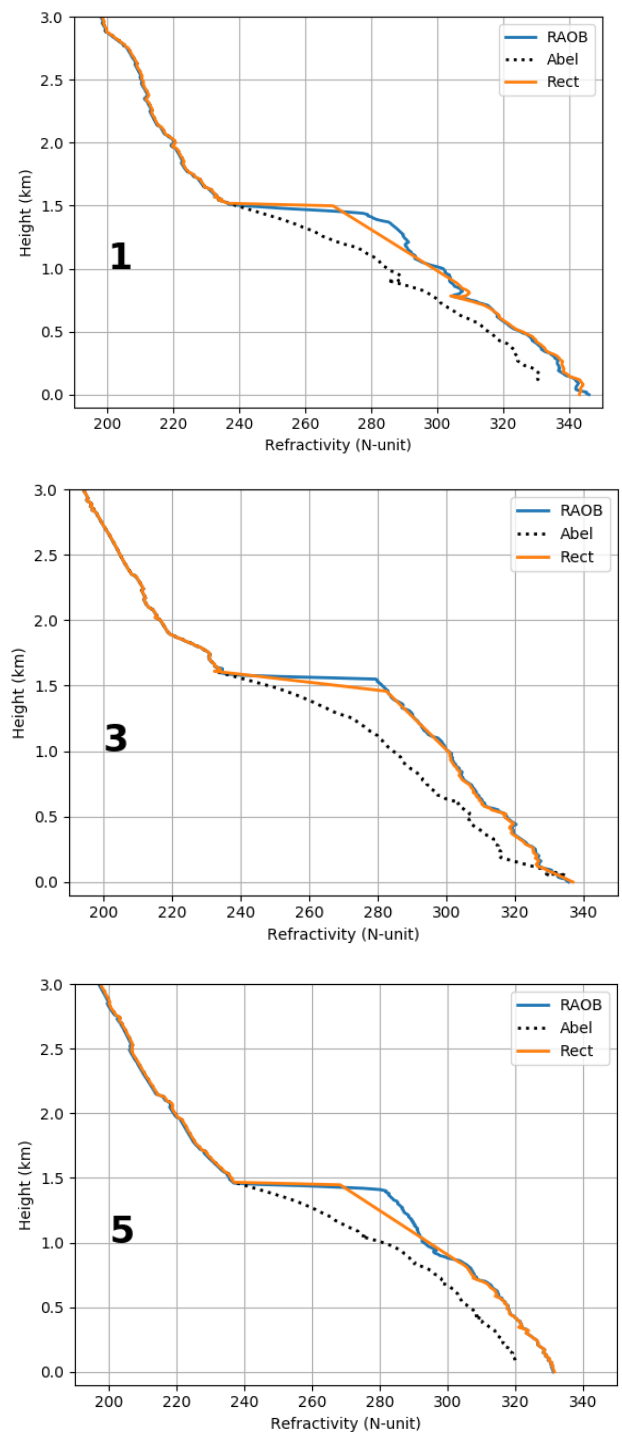
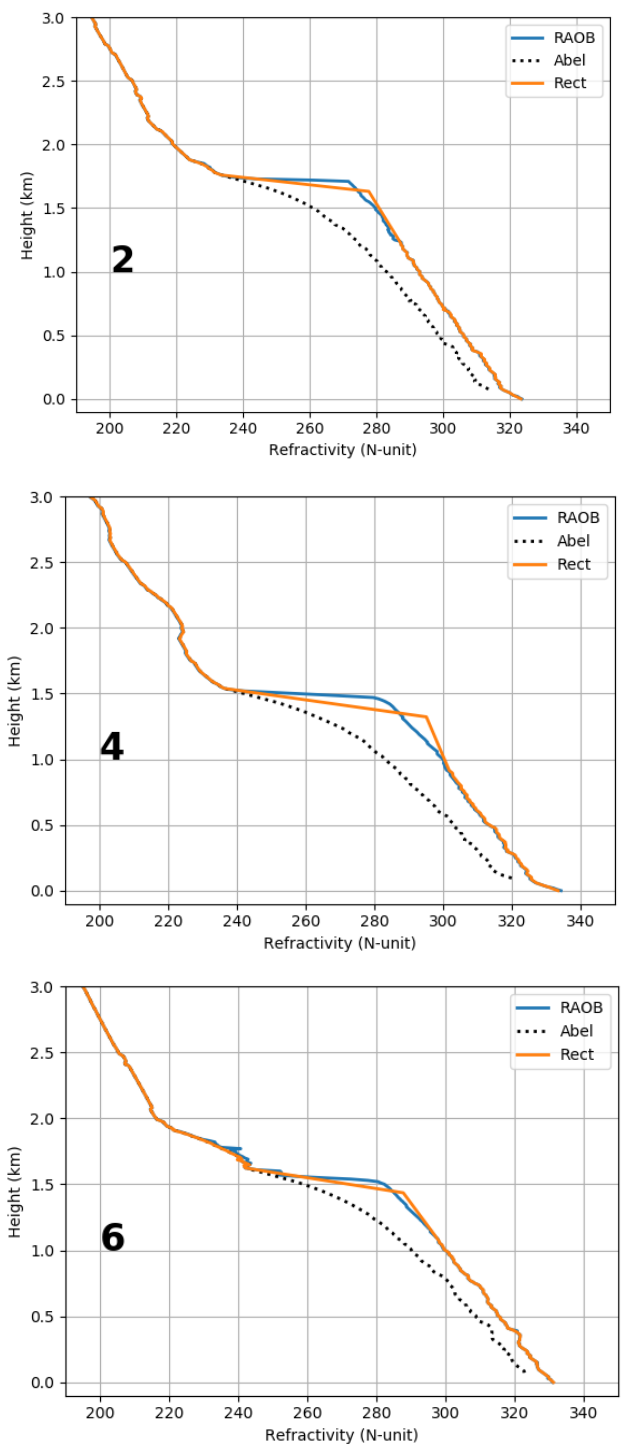

Figure 8. Refractivity profiles of simulated $\mathrm{RO}$, actual radiosonde measurements, and the reconstruction using the simulated reflected bending angle. These six cases are randomly chosen from the Variability of the American Monsoon Systems (VAMOS) Ocean-Cloud-Atmosphere-Land Study (VOCALS) campaign. As the figure shows, optimal estimation can correct the N-bias below ducting (where the refractivity profiles increase sharply at $\sim 1.5 \mathrm{~km}$ ) with the known reflected bending angle. 
The statistical reconstruction results compared to the RAOB observations are given in Figure 11. The refractivity difference in percentage in the horizontal axis is defined as:

$$
\Delta N(h)=\frac{N(h)-N_{R A O B}(h)}{N_{R A O B}(h)} \times 100 \%,
$$

where $N_{R A O B}(h)$ is the refractivity observation from RAOB as a function of height $h$, and $N(h)$ is either the results of Abel-inversion (black, dotted lines) or the reconstruction (orange, solid lines). As Figure 11 shows, the least squares reconstruction can decrease the $\mathrm{N}$-bias from $5 \%$ in Abel inverse profiles to less than $1 \%$ at $0.5 \mathrm{~km}$ below the top of the ducting layer. The large difference between the reconstruction results and the RAOB observations between $h_{t}-0.5 \mathrm{~km}$ to $h_{t}$ is typically due to mis-modeling inside the ducting layer, but this error does not have much influence on the reconstruction below.
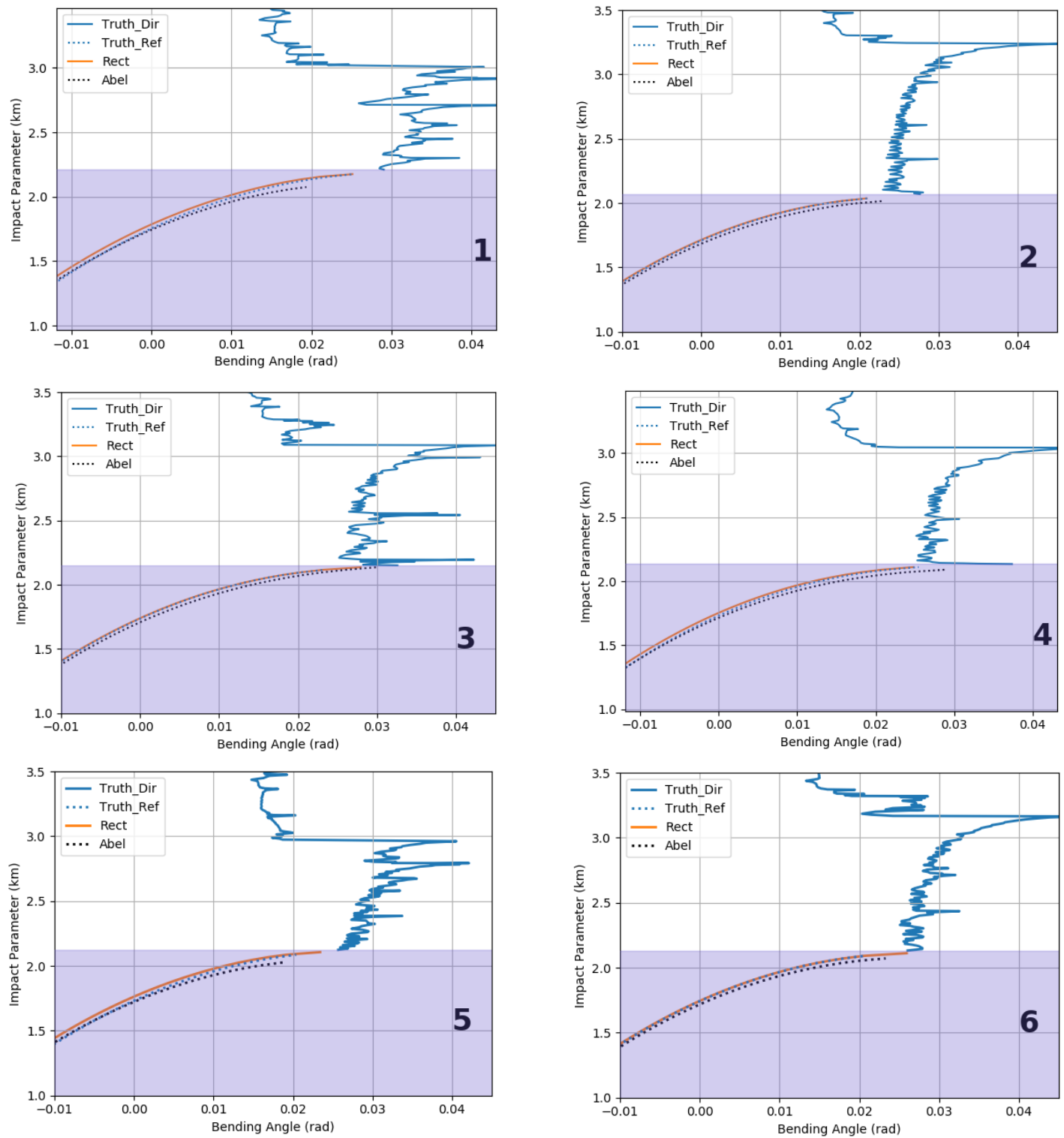

Figure 9. Corresponding reflected bending angle of each case shown in Figure 8. Since we choose the least difference in gradient, the resulting reflected bending angle is not necessarily aligned to the one calculated by forward Abel. 

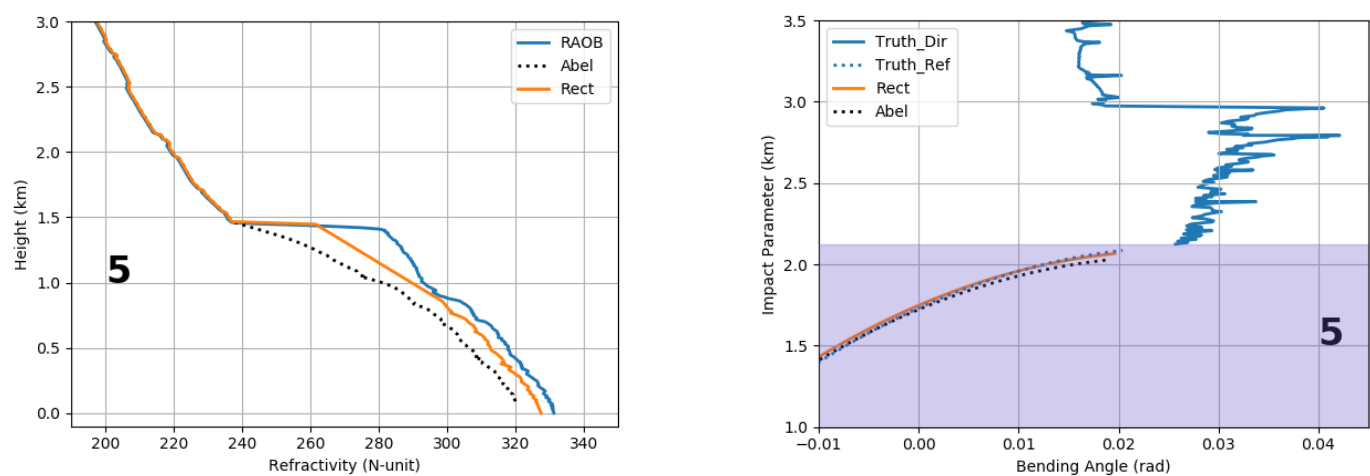

Figure 10. Same case (case 5) as shown in Figure 9 but using bending angle difference, rather than bending gradient difference, as the cost function. Underestimated $x_{m}$ is chosen, and the estimated result is still negatively biased compared to the true profile.

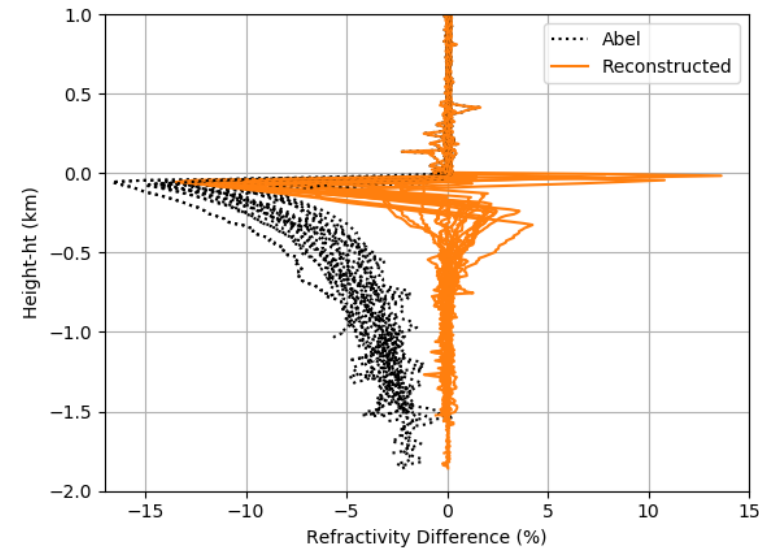

Figure 11. The refractivity differences between the radiosonde observations (RAOB) profiles, the Abel-retrieved profiles, and the reconstructed profiles for the 20 simulation cases with the single ducting layer in the VOCALS campaign, using the reflected bending angle from RAOB.

\subsection{Actual RO Cases}

In practice, the reflected bending angle retrieval is not straightforward. The reflected bending angle $\alpha_{R}(a)$ extracted by PM can be a multi-valued function in the impact parameter domain due to a horizontally non-uniform atmosphere [30]. In addition, the smoothing of the reflected bending angle over the impact parameter in PM can "shift" the profile vertically because the reflected bending angle has strong gradient close to the surface. To overcome the difficulty, the conventional GO processing for bending angle calculation in the time domain after removing the direct ray path component in the impact parameter domain is a more favorable approach [30]. According to Gorbunov et al. [30], the reflected RO ray is monotonic in the time domain because of the large rate of the grazing angle increasing and is immune to the multi-path effect, which is commonly observed in the direct RO signals over the lower troposphere. Therefore, PM is not essential to the reflected bending angle retrieval in practice, and it is only used in this research as a tool to remove the direct signal in the impact parameter domain. The details of the reflected signal extraction using $\mathrm{PM}$ and the application of inverse PM to transform the signal back to time domain for conventional GO processing is described in Appendix $\mathrm{C}$.

The GO algorithm calculating the reflected bending angle is the same as the one used for direct signal described in Gorbunov et al. [30]. The processing is demonstrated by an example with a strong reflection component, as shown in Figure 12a. Compared to its corresponding collocated European Centre for Medium-Range Weather Forecast (ECMWF) model, the RO retrieval shows a large negative bias, which indicates this occultation event is impacted by ducting. By applying the filtering process 
described in Appendix $C$, the direct signal is removed and the component left in the spectrogram (Figure 12b) is the extracted reflected signal in the time domain. The resulting amplitude and the excess Doppler example of the reflected signal in the time domain are shown in Figure 12c,d. The blue lines are for full signal, and the orange lines are the reflected signal only. The excess Doppler of full and reflected signals align to each other at the end of setting occultation because the ray path difference is decreasing through the time. Note that, while the reflected SNR reaches $40 \mathrm{~V} / \mathrm{V}$ in average for this specific case, in most other ducting cases, it is below $30 \mathrm{~V} / \mathrm{V}$.

Here, we hand-picked four actual RO cases with a clear reflected signal component in their spectrogram from the Radio Occultation Meteorology Satellite Application Facility (ROM SAF) reflection flag database [34]. All four cases are located in the north-east Pacific Ocean between September and November, where the strong inversion caused stratocumulus deck is frequently observed. The excess Doppler is then used in GO to calculate the reflected bending angle, which is shown in Figure 13 as the blue, dashed line. To suppress the noise, a 1-s window moving average on both the reflected bending angle and its corresponding impact parameter is applied. Only the portion where $0.005 \mathrm{rad}<\alpha_{R}<0.015 \mathrm{rad}$ is used as the measurement in this research to avoid the erroneous estimation due to large fluctuation. The best-matched profiles can be found using least squares, shown as the orange lines in Figure 14, and their corresponding reflected bending angle is shown as the orange line in Figure 13. The collocated ECMWF profiles are provided for comparison. It can be observed that the chosen critical height $h_{b}$ in the reconstructed profiles may be different from the ones that shown in ECMWF profiles, which could be due to the low bias of ECMWF model. But, as shown in the results, the reconstructed profiles significantly reduce the bias observed in original RO profiles (black, dashed line) and closer to the referenced ECMWF (blue, solid line) profiles.

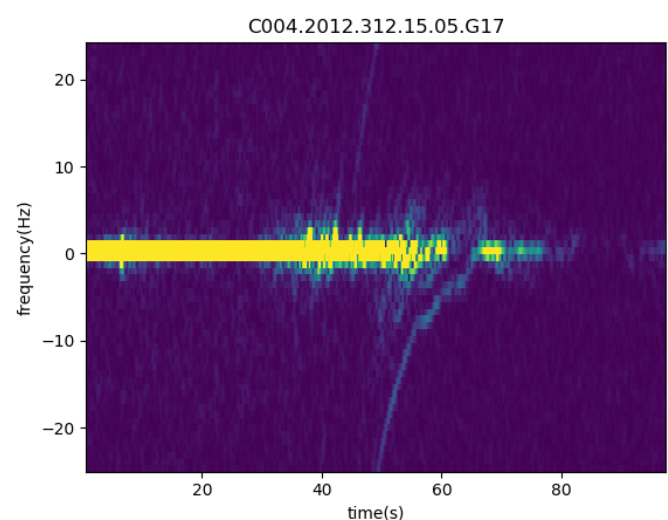

(a)

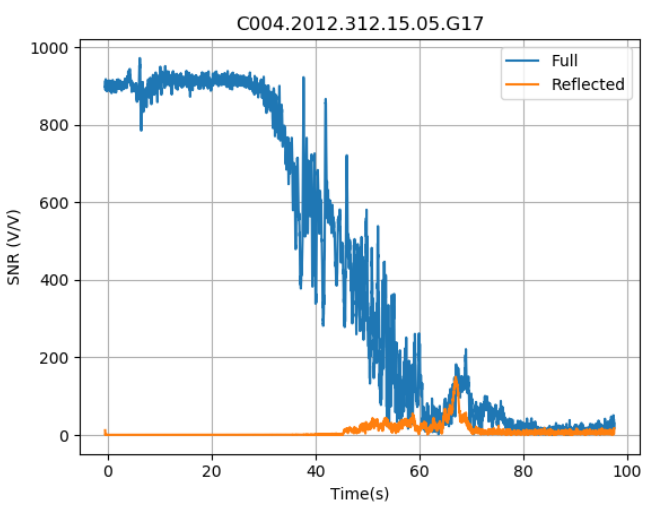

(c)

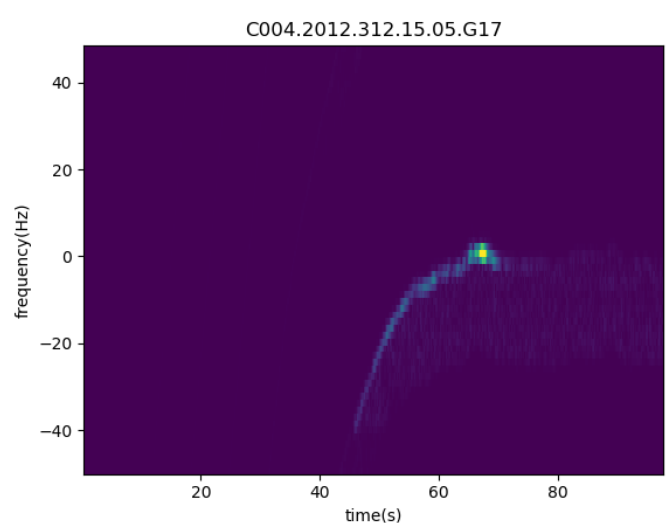

(b)

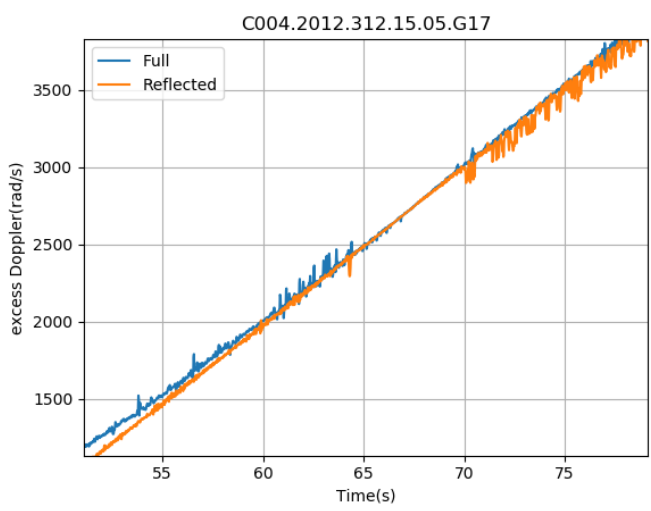

(d)

Figure 12. Spectrogram example of an actual RO case: (a) before (b) after RO filtering. The reflection component can be extracted after the RO filtering is applied. The signal-to-noise ratio (SNR) and the excess phase of the full and reflected signal are shown in $(\mathbf{c}, \mathbf{d})$. 
While the unbiased candidate profiles can be identified in these cases, it is not common for the reflected signals to reach such a high SNR. In fact, the resulting reflected bending angle, in most of the cases calculated by GO, contains significant fluctuation, which makes applying least-squares estimation difficult. This large variance of the bending angle is due to the low SNR nature of the reflected signal caused by the delay offset and frequency divergence from the local generated signal, as well as the interference from the direct signal at the end of the setting occultations (or the beginning of the rising occultations). To quantify the uncertainties in the reflected bending calculation, the spectral width in the impact parameter is shown in Figure 13 as the shaded area. The calculation of the uncertainty for this specific case is provided by Gorbunov et al. [35]. It can be observed that the bending angle could contain the impact parameter uncertainty of $0.2 \mathrm{~km}$ and noticeably higher when its corresponding impact parameter is close to the surface. This big uncertainty can, therefore, jeopardize the proposed reconstruction method, and possible improvements are proposed in the discussion section.
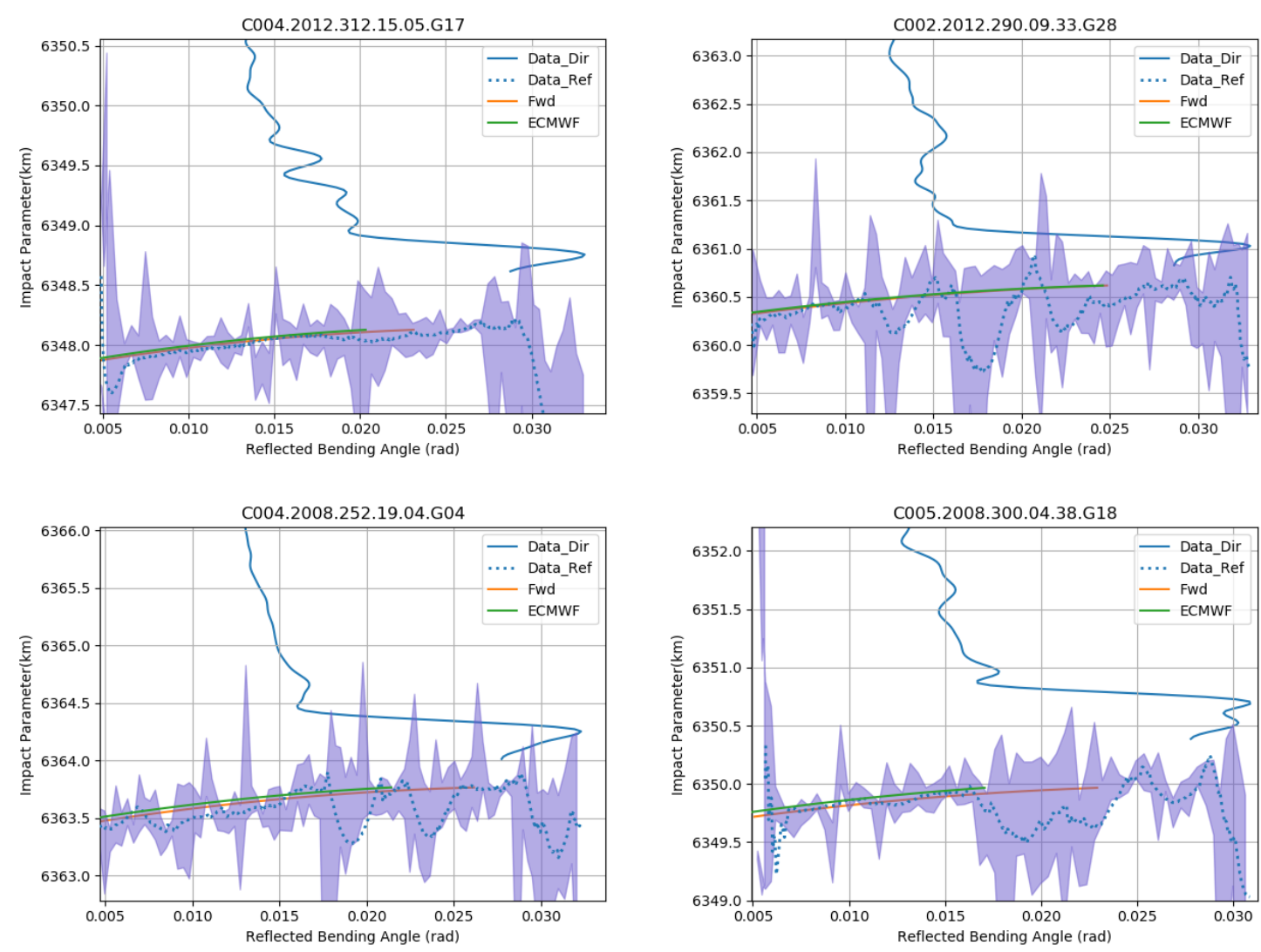

Figure 13. Correction results of the actual case: BA profiles. The blue dashed lines are reflected bending angle observations calculated with GO, and the shaded area is the uncertainty that can be related to SNR. The chosen candidate (orange color) is close to the European Centre for Medium-Range Weather Forecast (ECMWF) forwarded bending angle (green color) in most of the cases. 

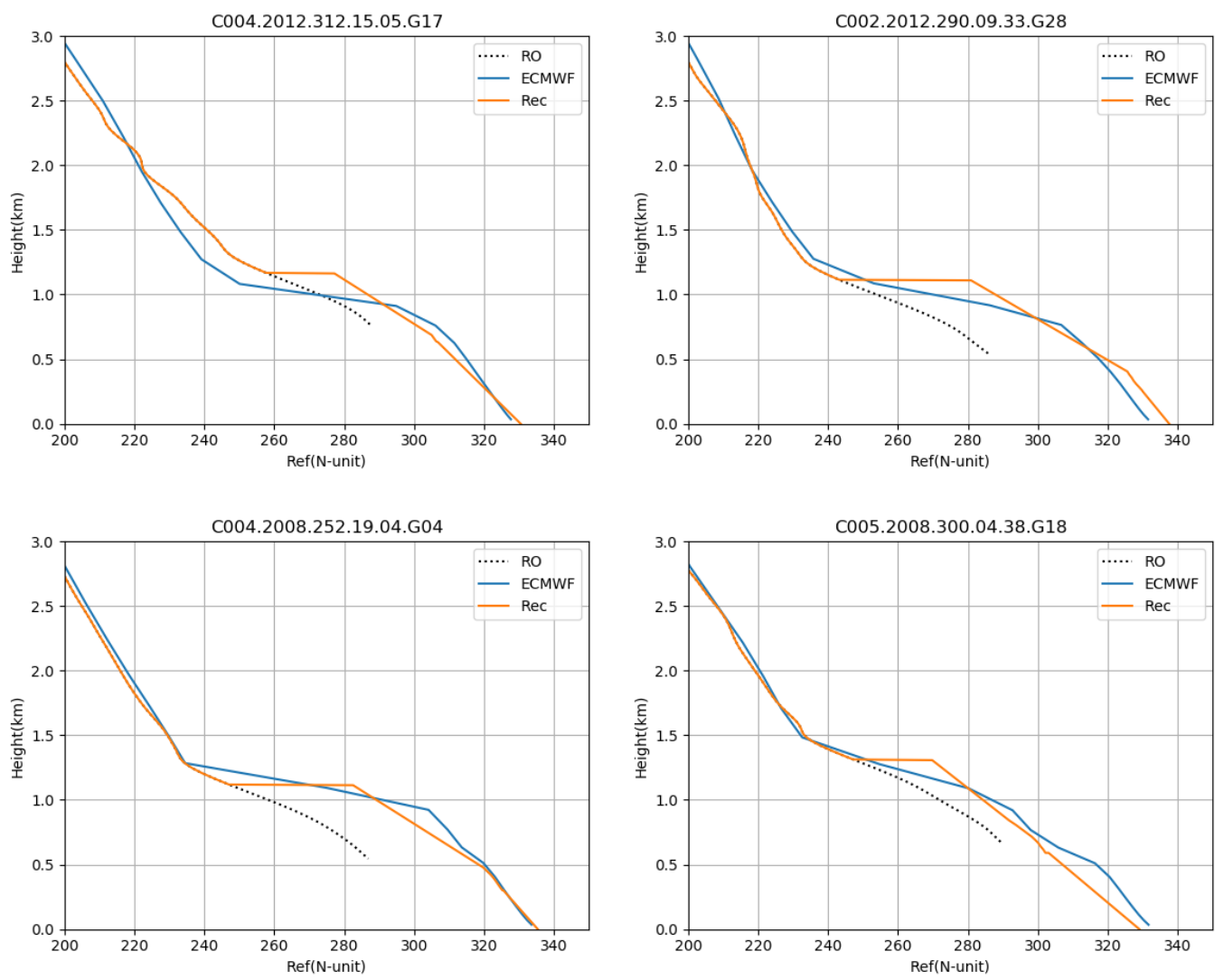

Figure 14. Correction results of the actual case: $\mathrm{N}$ profiles. The reconstructed result (orange color) using reflected bending angle can successfully correct the $N$-bias in Abel-inverse profile (black color, dashed line) compared to the collocated ECMWF model and RAOB observations.

\section{Discussion}

As shown in the previous section, using the constraint of the reflected bending angle can effectively reduce the $\mathrm{N}$-bias caused by ducting. While this approach significantly enhanced the usability of the $\mathrm{N}$-bias correction algorithm in practice, some limitations do exist. First, this reconstruction method does not provide additional information of the missing structure that ordinary GNSS-RO cannot detect, such as the profile inside the ducting layer and below the penetration depth. The $x-h$ structure inside the ducting layer and the refrativity profile below the RO cut-off height is provided by the bilinear shape and exponential extension assumption, respectively, rather than retrieved from the RO measurements. Therefore, the error in the reconstruction results can show up due to the erroneous modeling of the thermodynamic structure. While the relationship between them is not straightforward and the sensitivity is unknown, the reflected bending angle can potentially provide the surface refractivity information and the physical constraint inside the ducting layer. Thus, through the better reflected bending angle processing, more information could possibly be extracted and enhance the reconstruction in the future.

Another limitation is the low signal strength of the reflected signal. Although the amplitude information is not directly used in the reconstruction algorithm, low SNR could cause a large variance in the retrieved reflected bending angle. As mentioned above, the SNR of the reflected signals is lower because, in the current open-loop tracking receiver, the local carrier frequency and delay correlator are set to track the direct signal. Therefore, the reflected signal strength decreases when its path length has a larger difference than the direct path length (i.e., higher tangent point altitude). The other possible cause is the signal strength loss due to the sea surface roughness $(g)$, which can be quantified by the Rayleigh roughness parameter [36]: 


$$
g=4 \pi\left(\frac{S_{h}}{\lambda}\right) \sin \phi,
$$

where $S_{h}$ is the standard deviation of the surface height about the local mean, $\lambda$ is the signal wave length, and $\phi$ is the grazing angle. Equation (16) shows that the small grazing angle significantly decreases $g$ to less than 0.5 , which eases the roughness problem. The corresponding reduction factor of the specular component can be modeled [36] as:

$$
\rho_{s}=\exp \left(-1 / 2 g^{2}\right)
$$

where $\rho_{s}$ is the reduction factor of the reflected signal amplitude. The average global sea surface wind speed is around $6 \mathrm{~m} / \mathrm{s}$, which could statistically lead to $1 \mathrm{~m}$ significant wave height $\left(S_{h}=0.25\right)$ over a fully developed sea [37]. In the reflection case of $-300 \mathrm{~m}$ tangent point height with grazing angle of $1 \mathrm{e}-3 \mathrm{rad}, \rho_{s}=0.987$ and causes only less than $2 \%$ of signal amplitude attenuation. Even on a rough sea surface of $3 m$ significant wave height, $\rho_{s}=0.89$ and still causes only $\sim 1 / 10$ of amplitude loss. Therefore, only in the most extreme cases could the surface roughness negatively impact the proposed $N$-bias correction algorithm.

Due to the SNR limitation, the fraction of the high reflected SNR cases among the reflection-detected occultation suitable for the reconstruction algorithm may be considerably less than $45 \%$ in mid-latitude regions. This requires further investigations. But the low SNR issue can be overcome in two ways. First, the reflected SNR can be enhanced if the total SNR increases. The SNR of the recently launched COSMIC-2 mission can reach $2500 \mathrm{~V} / \mathrm{V}$ with the newly designed beam-forming antenna compared to an average of $700 \mathrm{~V} / \mathrm{V}$ in the current COSMIC mission. Hence, the reflected SNR can be significantly improved, and a higher percentage of RO cases that the proposed method can be applied is expected. The other possible solution is to modify the receiver operation to track the reflected signal with a separate RF channel. The advantage of this approach is that no further processing to extract the reflected signal (Appendix C) is required, which can simplify and improve the reflected signal processing on the ground. These improvements can effectively enhance the reflected SNR and ease the application of the proposed reconstruction method in practice.

\section{Conclusions}

GNSS-RO is a valuable measurement technique for probing the vertical thermodynamic structure of the PBL from space [6]. However, it is known that a negative refractivity bias ( $N$-bias) can exist within PBL, which will lead to retrieved humidity and temperature biases. Over the stratocumulus regon in the subtropical oceans, the $N$-bias is dominated by the prevalence of elevated ducts caused by the strong inversion capping the PBL $[14,15]$. To improve GNSS-RO's capability of characterizing PBL structure, correcting the N-bias within the PBL caused by ducting is critical. Due to the under-determined condition in standard Abel inversion, the $N$-bias generated by strong refractivity gradient $(<-157 \mathrm{Nunit} / \mathrm{km})$ can reach 5\% within the PBL. In previous studies [18,23], the analytical solutions based on the biased refractivity profile and the physical constraints, including the RO measurements at the surface or external information (e.g., precipitable water) from other collocated observations, are derived. However, actual RO measurements rarely reach the surface, and collocation between GNSS-RO and other observations does not always exist. In addition, the use of external information makes the solution dependent on these data, which may contain biases themselves.

In this article, a new bias reduction method is introduced using the reflected signal component during the occultation events. The grazing components were extracted from the received RO signals, and the bending angle of their ray paths was used as the constraint to solve the ill-posed inversion problem. We implemented the least square algorithm to select the candidate profile that best matches the observed reflected bending gradient from the solution family. The significant advantage of this method is that it does not rely on external information; thus, it eliminates possible errors arising from 
the assisting observations or models. Based on the simulation and actual RO data results, the proposed reconstruction method is able to reduce the $N$-bias to less than $1 \%$ below the ducting layer. The new construction method has also been applied to the actual $\mathrm{RO}$ data: the resulting solutions removed most of the N-bias and are consistent with the collocated model profile. While several limitations, including the low SNR and surface characteristics, could affect the applicability of the proposed method, the challenges can be overcome with the higher SNR and the better grazing RO signal tracking technique in future $\mathrm{RO}$ missions.

Author Contributions: K.-N.W., C.O.A., and M.d.I.T.J. conceptualized the method. K.N.W. developed the methodology, implemented the software, and perform the testing and validation. C.O.A. supervised the project. K.-N.W. and C.O.A. wrote the manuscript. All authors have read and agreed to the published version of the manuscript.

Funding: This research was funded by NASA ROSES Grant No. 14-GNSS14-0017.

Acknowledgments: The research described in this paper was carried out at the Jet Propulsion Laboratory (JPL), California Institute of Technology, under a contract with the National Aeronautics and Space Administration. We would like to thank European Centre for Medium-Range Weather Forecasts (ECMWF) for providing the high resolution gridded analysis profiles and NCAR EOL for radiosonde data access.

Conflicts of Interest: The authors declare no conflict of interest.

\section{Appendix A. Family Solution for RO Refractivity Profiles in Ducting Condition}

Xie et al. [18] showed that the infinite number of refractivity profiles connect to the same direct bending angle through Abel inversion can be mathematically described when the profile $h(x)$ can be approximated as bilinear structure inside the ducting layer:

$$
\begin{aligned}
& h_{2}(x)=h_{b}+\frac{h_{m}-h_{b}}{x_{m}-x_{b}}\left(x-x_{b}\right), \\
& h_{3}(x)=h_{t}-\frac{h_{t}-h_{m}}{x_{m}-x_{b}}\left(x-x_{b}\right) .
\end{aligned}
$$

Under this assumption, the profile below the ducting layer can then be analytically written as:

$$
h_{1}(x)=h_{A}(x)+\frac{2}{\pi}\left(h_{t}-h_{b}\right)\left[z-\left(1+z^{2}\right) \tan ^{-1}(1 / z)\right],
$$

where $h_{A}(x)$ is the height function with respect to $x$ from the standard Abel refractivity retrieval (grey curve in Figure $1 b$ ), and $z=\sqrt{x_{b}-x} / \sqrt{x_{m}-x_{b}}$. By using Equation (A3), all the candidate refractivity profile solutions, can be produced when the five parameters defining the ducting layer structure: $x_{b}, x_{m}$, $h_{b}, h_{m}$, and $h_{t}$, are given. In this paper, $x_{b}$ can be acquired by using the correlation method proposed by Wang et al. [23], and the height it intersects with $h_{A}(x)$ is $h_{t} . x_{m}$ is an independent parameter that is to be determined by the least square optimization. $h_{b}$ and $h_{m}$ can be calculated based on each different candidate $x_{m}$, where several constraints are applied. Here, the $h_{b}$ is determined as the value that causes the minimum RMS residual when taking linear regression on the resulting $h_{1}(x)$ between $x_{b}$ and $x_{b}-0.2$ $\mathrm{km}$, which is proposed by Xie et al. [18]. Then, the $h_{1}(x)$ is acquired, and the $h_{m}$ can be calculated simply by extending the straight fitting line between $x_{b}$ and $x_{b}-0.2 \mathrm{~km}$ to the designated $x_{m}$. Compared to the approach used in Wang et al. [23], this modification provides a conservative though robust solution for each parameter's combination to further remove the spikes and the unreasonable profiles due to the high sensitivity of Equation (A3) to the mis-modeling inside the ducting layer.

\section{Appendix B. Sensitivity Test of the Exponential Extrapolation}

In general, GNSS-RO profile does not reach the surface due to low SNR and high refractivity gradient at the lower troposphere. As a result, the refractivity profile needs to be extrapolated down to the surface to calculate the reflected bending angle with Equation (8). Here, we provide two sensitivity tests of different (1) truncation height and (2) extrapolation implementation to understand 
how the RO penetration and profile extrapolation can affect the reflected bending angle and the reconstructed results.

The same truth profile described by Equation (1) is utilized. To simulate the different RO penetration depths, we firstly removed the bottom $0,0.2,0.4,0.6,0.8 \mathrm{~km}$ of the biased refractivity profiles that were calculated by Equations (3) and (4). These five profiles are then be used as the input of the reconstruction method described in Section 3.1. Here, the bottom portion (lowest $0.4 \mathrm{~km}$ ) of each iterative candidate was taken to fit the exponential functions and extrapolate the profile down to the surface. The reconstruction results of these five profiles are shown in Figure A1a, where the left panel shows the reconstructed and the Abel-inverse $N$-profiles, and the right panel shows their resulting error compared to the true profile simulated by Equation (1). The figure shows that the reconstruction is not very sensitive to the penetration height in this case, probably due to the fact that the original equation of the profile (Equation (1)) is also modified from an exponential function. The error mostly occurs at the height of the ducting and shadow layer $(1.5-2 \mathrm{~km})$ and is significantly reduced below. This is because the least square reconstruction using reflecting bending angle gradient tends to choose the profiles that have the correct surface impact parameter and gradually puts less weight on the structure above. However, it still can be seen that lower truncation height reconstructed profiles have less error compared to the truth profile in the right panel of Figure A1a. Therefore, the decreasing extrapolation uncertainty can be expected with the higher SNR and lower penetration height of the coming new GNSS-RO missions.

The worst case in Figure A1a, the one truncated at $0.8 \mathrm{~km}$, is used here to illustrate the sensitivity of different extrapolations. In Figure A1b, this truncated profile has been reconstructed by fitting the lowest $0.24,0.36,0.48,0.6$, and $0.72 \mathrm{~km}$ with the exponential function. As the figure shows, the error at the ducting layer in the $0.72 \mathrm{~km}$ fitting case can exceed $-3 \%$. It is expected because the extrapolation by fitting the higher altitude could cause a large error around the surface. The error of all the other cases is well-maintained to less than $-1 \%$ below $1.5 \mathrm{~km}$. While the ducting layer cannot be better modeled due to limited information and the uncertainty caused by extrapolation that is difficult to control, the profiles below the ducting still show a great improvement compared to the Abel-inverse results. Note that no noise is added in this sensitivity test. In practice, a trade-off between the fitting interval and the uncertainty at the lowest part of the profile should be considered: A longer fitting interval could cause a larger error, but a shorter fitting interval could easily be affected by noisy refractivity observations at the bottom.
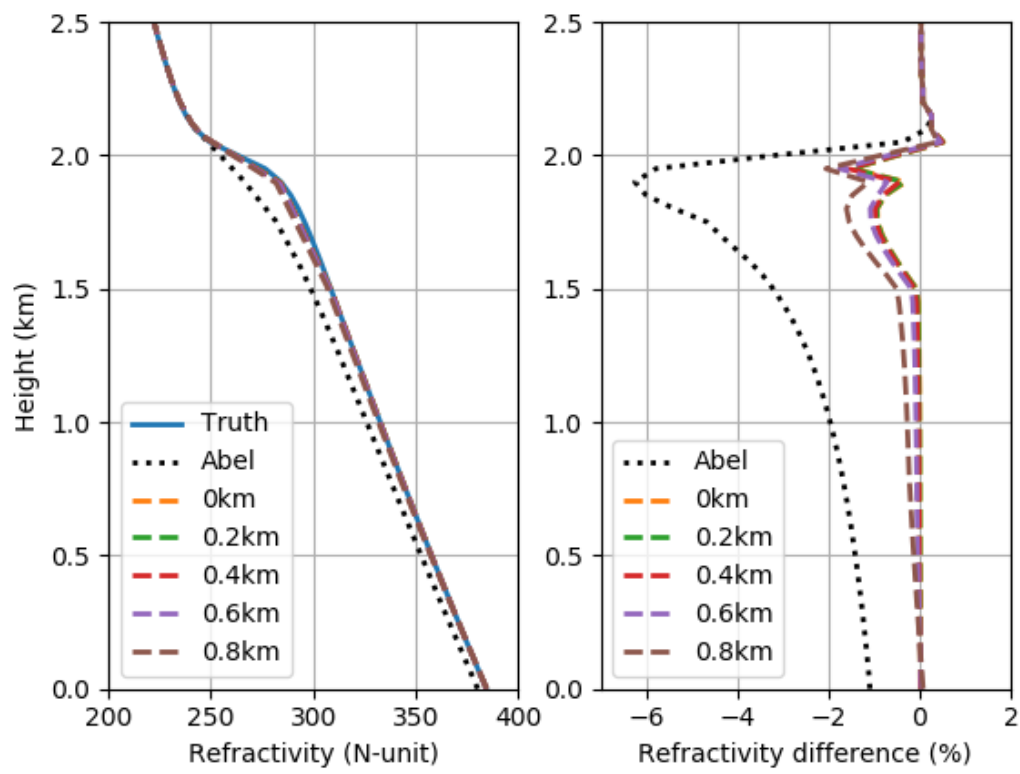

(a)

Figure A1. Cont. 

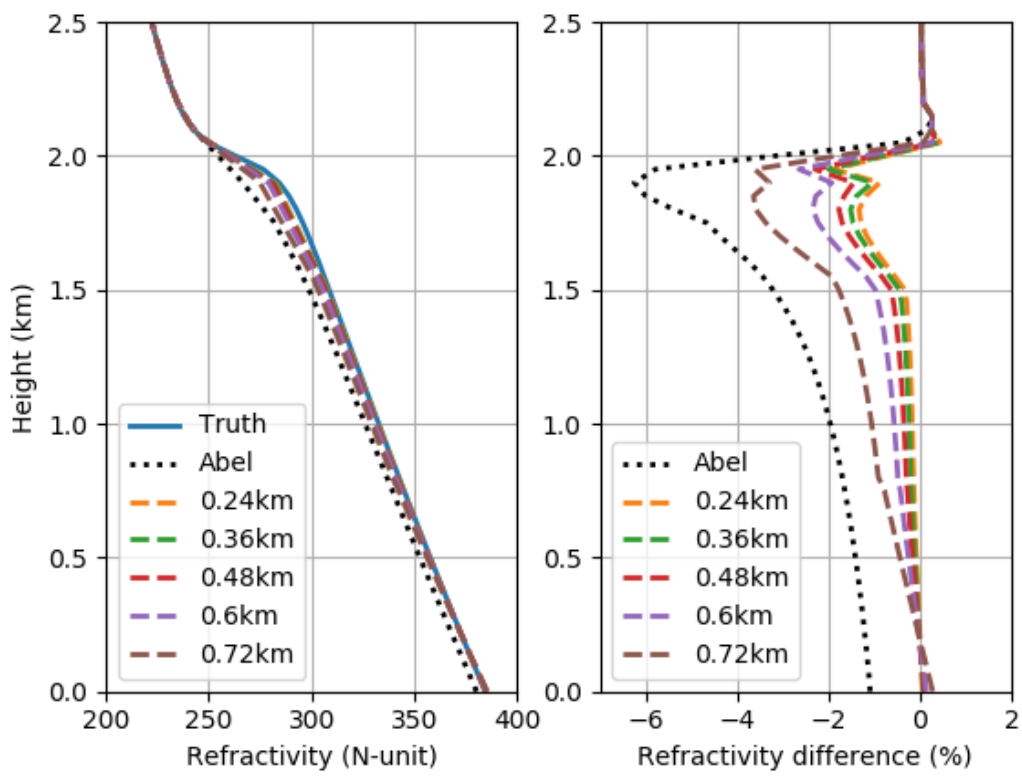

(b)

Figure A1. Sensitivity test of the refractivity profile extrapolation at the bottom layer. (a) The reconstructed refractivity error with different penetration height using the same fitting interval $(0.4 \mathrm{~km})$. (b) The reconstructed refractivity error with different extrapolation fitting interval under the same penetration height $(0.8 \mathrm{~km})$.

\section{Appendix C. Direct Signal Filtering Process}

In this section, we show how the received signal can be processed to remove the direct signal prior to obtaining the reflected bending angle using GO method. A similar approach as mentioned in Gorbunov et al. [30] is taken in this paper, whereas the PM method is used instead. Firstly, the phase of the complex received signal $s(t)=A(t) \exp [i \Phi(t)]$ has been smoothed with a 4-s window to get $\Phi_{s m}(t)$, where $\Phi(t)$ is the excess phase of the signal. The signal can then be down-shifted with respect to $\Phi_{s m}(t)$, similar to Equation (7):

$$
s^{\prime}(t)=\frac{s(t)}{\exp \left[i \Phi_{s m}(t)\right]}=A(t) \exp \left[i\left(\Phi(t)-\Phi_{s m}(t)\right)\right] .
$$

Since the smoothed phase mainly comes from the direct signal, the frequency down-shifting can be regarded as centering the signal at the direct path frequency. The spectrum of $s^{\prime}(t)$ is shown in Figure 12, where the reflected component can be clearly observed. Note that the reflection frequency components locate at both side of the spectrum due to the aliasing of $50 \mathrm{~Hz}$ sampling rate. To utilize the reflected signal at both ends, we separate $s^{\prime}(t)$ as follows:

$$
\begin{gathered}
s_{A}^{\prime}(t)=L P_{0-10 H z}\left[s^{\prime}(t)\right]=A_{A}(t) \exp \left[i \Phi_{A}(t)\right], \\
s_{B}^{\prime}(t)=L P_{0-15 H z}\left[s^{\prime}(t) \exp (i \cdot 2 \pi \cdot 25 t)\right]=A_{B}(t) \exp \left[i \Phi_{B}(t)\right],
\end{gathered}
$$

where $L P$ is the low-pass filtering operator with the bandwidth indicated in the subscript. $s_{A}^{\prime}(t)$ simply removes all the components with frequency $>10 \mathrm{~Hz}$ and that mainly contributed by the direct RO signal, while $s_{B}^{\prime}(t)$ shifts the center frequency by $25 \mathrm{~Hz}$ and preserves only the reflection part of the signal. Therefore, by ignoring the higher order term of the low pass filtering, the down-converted signal $s^{\prime}(t)$ can be roughly represented as:

$$
s^{\prime}(t) \cong s_{A}^{\prime}(t)+s_{B}^{\prime}(t) \exp (i \cdot 2 \pi \cdot-25 t) .
$$


The spectrum of both $s_{A}^{\prime}(t)$ and $s_{B}^{\prime}(t)$ are shown in Figure A2. As Figure A2b shows, the $25 \mathrm{~Hz}$ shifting of $s_{B}^{\prime}(t)$ connects the reflected frequency components in the center, which were located at the edge of both side of the spectrum, as shown in Figure 12. The new connected excess phase of these two complex signals, $\Phi_{A}^{\prime}(t)$ and $P h i_{B}^{\prime}(t)$, can then be calculated in the time domain by unwrapping $\Phi_{A}(t)$ and $\Phi_{B}(t)$ and add the $\Phi_{s m}(t)$ back in both channels.

$$
\begin{gathered}
\Phi_{A}^{\prime}(t)=U W\left[\Phi_{A}(t)\right]+\Phi_{s m}(t) . \\
\Phi_{B}^{\prime}(t)=U W\left[\Phi_{B}(t)\right]+\Phi_{s m}(t)-2 \pi 25 t,
\end{gathered}
$$

where UW is the unwrapping operator. Note that the $25 \mathrm{~Hz}$ component that was added in Equation (A6) is removed here in $\Phi_{B}^{\prime}(t)$. In this way, the non-aliased reflected component is preserved in the time domain excess phase. The two signals can then be combined as:

$$
s^{\prime \prime}(t)=A_{A}(t) \exp \left[i\left(\Phi_{A}^{\prime}(t)+\Phi_{G E O}(t)\right)\right]+A_{B}(t) \exp \left[i\left(\Phi_{B}^{\prime}(t)+\Phi_{G E O}(t)\right)\right] \text {. }
$$

Here, we add the geometric phase $\Phi_{G E O}(t)$, which equals to the geometric distance between the transmitter and the receiver, to the excess phase term to calculate the total phase for each sub-signal. Both $\Phi_{A}^{\prime}(t)$ and $\Phi_{B}^{\prime}(t)$ will be up-sampled as required by PM at this step, which also prevents aliasing of the reflected signal. Then, the processed signal can be transformed to the impact parameter domain by performing PM [38]:

$$
S(a)=\int s^{\prime \prime}(t) \exp [-i k \Psi(a, t)] d t
$$

where $k$ is the wave number, $a$ is the impact parameter, and $\Psi(a, t)$ is the modeled phase function of the parameter $a$ and time $t$. The spectrum of $S(a)$ is shown in Figure A3. Since the aliasing portion has been moved below, no extra reflected component will show up at about $8 \mathrm{~km}$ above the surface, as stated in Gorbunov et al. [30]. Therefore, we can simply remove all of the component above the surface in the impact parameter domain, i.e., direct signal, and transform back to the time domain with inverse PM:

$$
s_{R}(t)=\int S(a) f(a) \exp [i k \Psi(a, t)] d a,
$$

where $f(a)$ is the filtering function of impact parameter $a$ to remove the direct signal. In practice, we determine the surface impact parameter $a_{0}$ by applying the correlation method on the bending angle profile, as described in the appendix of Wang et al. [23], while the reversed step function is used; therefore, no information from the model is needed. The value of the function $f(a)$ will then be set as 0 when $a>a_{0}$ and as 1 when $a<a_{0}$ for extracting the reflected signal. The resulting $s_{R}(t)$ is the pure reflected signal as a function of time, which can be used later in GO to obtain the reflected bending angle. Note that $s_{R}(t)$ is calculated in the sample. For this specific case, the spectrum of $s_{R}(t)$ is shown in Figure 12.

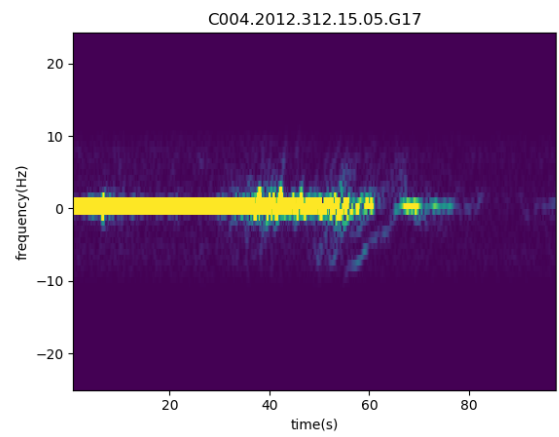

(a)

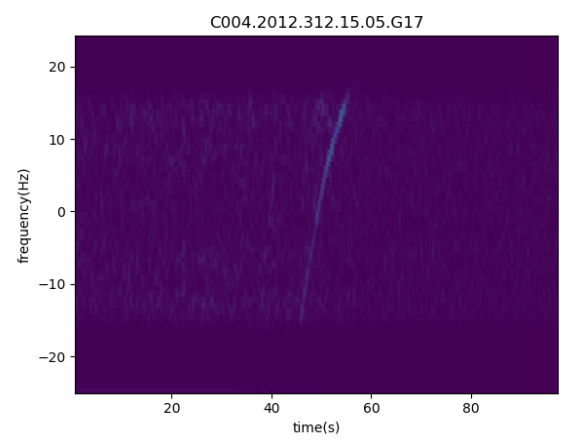

(b)

Figure A2. The spectrogram of the sub-signal (a) $s_{A}^{\prime}(t)$ and (b) $s_{B}^{\prime}(t)$. The $s_{B}^{\prime}(t)$ has been shifted $25 \mathrm{~Hz}$ to connect the reflected component at both side of the spectrum. 


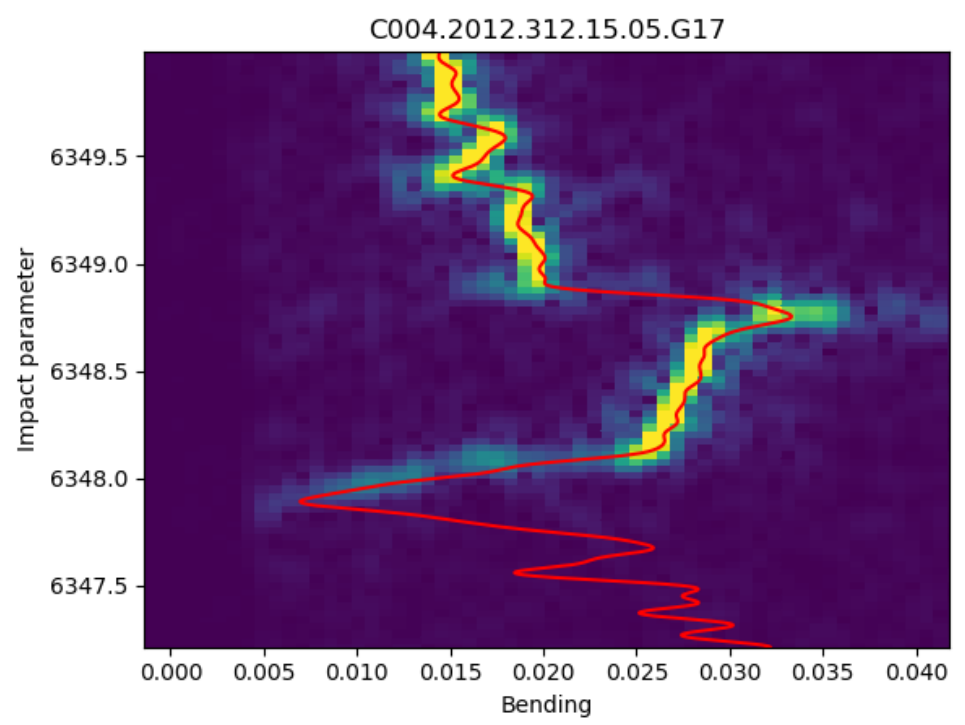

Figure A3. The spectrogram of the signal $S(a)$ onto impact parameter domain. Reflected component is the one distributed horizontally at $6348 \mathrm{~km}$. Because the reflected components have been connected in the frequency domain, the aliasing portion will not be shown in the figure. The red line is the bending angle results from the phase matching method.

\section{References}

1. Kursinski, E.R.; Hajj, G.A.; Schofield, J.T.; Linfield, R.P.; Hardy, K.R. Observing Earth's atmosphere with radio occultation measurements using the Global Positioning System. J. Geophys. Res. 1997, 102, 23429-23466. [CrossRef]

2. Anthes, R.; Schreiner, W. Six new satellites watch the atmosphere over Earth's equator. EOS Earth Space Sci. News 2019. Available online: https: / / eos.org/science-updates/six-new-satellites-watch-the-atmosphereover-earths-equator (accessed on 10 January 2020).

3. Sokolovskiy, S.; Rocken, C.; Lenschow, D.H.; Kuo, Y.-H.; Anthes, R.A.; Schreiner, W.S.; Hunt, D.C. Observing the moist troposphere with radio occultation signals from COSMIC. Geophys. Res. Lett. 2007, 34, L18802. [CrossRef]

4. von Engeln, A.; Teixeira, J.; Wickert, J.; Buehler, S.A. Using CHAMP radio occultation data to determine the top altitude of the Planetary Boundary Layer. Geophys. Res. Lett. 2005, 32, L06815. [CrossRef]

5. Garratt, J.R. The Atmospheric Boundary Layer; Cambridge University Press: Cambridge, UK, 1992; p. 334.

6. National Academies of Sciences, Engineering, and Medicine. Thriving on Our Changing Planet: A Decadal Strategy for Earth Observation from Space; The National Academies Press: Washington, DC, USA, 2018. [CrossRef]

7. Gorbunov, M.E.; Benzon, H.-H.; Jensen, A.S.; Lohmann, M.S.; Nielsen, A.S. Comparative analysis of radio occultation processing approaches based on Fourier integral operators. Radio Sci. 2004, 39, RS6004. [CrossRef]

8. Curran, R.J. Satellite-borne lidar observations of the Earth: Requirements and anticipated capabilities. Proc. IEEE 1989, 77, 478-490. [CrossRef]

9. Solheim, F.S.;Vivekanandan, J.; Ware, R.H.; Rocken, C. Propagation delays induced in GPS signals by dry air, water vapor, hydrometeors, and other particulates. J. Geophys. Res. 1999, 104, 9663-9670. [CrossRef]

10. Ao, C.O.; Waliser, D.E.; Chan, S.K.; Li, J.-L.; Tian, B.; Xie, F.; Mannucci, A.J. Planetary boundary layer heights from GPS radio occultation refractivity and humidity profiles. J. Geophys. Res. 2012, 117, D16117. [CrossRef]

11. Chan K.M.; Wood, R. The seasonal cycle of planetary boundary layer depth determined using COSMIC radio occultation data. J. Geophys. Res. 2013, 118, 12,422-12,434. [CrossRef]

12. Guo, P.; Kuo, Y.-H.; Sokolovskiy, S.V.; Lenschow, D.H. Estimating atmospheric boundary layer depth using COSMIC radio occultation data. J. Atmos. Sci. 2011, 68, 1703-1713. [CrossRef] 
13. Ho, S.-P.; Peng, L.; Anthes, R.A.; Kuo, Y.-H.; Lin, H.-C. Marine Boundary Layer Heights and Their Longitudinal, Diurnal, and Interseasonal Variability in the Southeastern Pacific Using COSMIC, CALIOP, and Radiosonde Data. J. Clim. 2015, 28, 2856-2872. [CrossRef]

14. Xie, F.; Wu, D.L.; Ao, C.O.; Mannucci, A.J.; Kursinski, E.R. Advances and limitations of atmospheric boundary layer observations with GPS occultation over southeast Pacific Ocean. Atmos. Chem. Phys. 2012, 12, 903-918. [CrossRef]

15. von Engeln, A.; Teixeira, J. A ducting climatology derived from the European Centre for Medium-Range Weather Forecasts global analysis fields. J. Geophys. Res. 2004, 109, D18104. [CrossRef]

16. Ao, C.O. Effect of ducting on radio occultation measurements: An assessment based on high-resolution radiosonde soundings. Radio Sci. 2007, 42. [CrossRef]

17. Sokolovskiy, S. Effect of superrefraction on inversions of radio occultation signals in the lower troposphere. Radio Sci. 2003, 38, 1058. [CrossRef]

18. Xie, F.; Syndergaard, S.; Kursinski, E.R.; Herman, B.M. An approach for retrieving marine boundary layer refractivity from GPS occultation data in the presence of superrefraction. J. Atmos. Ocean. Technol. 2006, 23, 1629-1644. [CrossRef]

19. Ao, C.O.; Meehan, T.K.; Hajj, G.A.; Mannucci, A.J.; Beyerle, G. Lower troposphere refractivity bias in GPS occultation retrievals. J. Geophys. Res. 2003, 108, 4577., D18. [CrossRef]

20. Sokolovskiy, S. Tracking tropospheric radio occultation signals from low Earth orbit. Radio Sci. 2001, 36, 483-498. [CrossRef]

21. Sokolovskiy, S.; Rocken, C.; Schreiner, W.S.; Hunt, D.C. On the uncertainty of radio occultation inversions in the lower troposphere. J. Geophys. Res. 2010, 115, D22111. [CrossRef]

22. Gorbunov, M.E.; Vorob'ev, V.V.; Lauritsen, K.B. Fluctuations of refractivity as a systematic error source in radio occultations. Radio Sci. 2015, 50, 656-669. [CrossRef]

23. Wang, K.-N.; de la Torre Juárez, M.; Ao, C.O.; Xie, F. Correcting negatively biased refractivity below ducts in GNSS radio occultation: An optimal estimation approach towards improving planetary boundary layer (PBL) characterization. Atmos. Meas. Tech. 2017, 10, 4761-4776. [CrossRef]

24. Aparicio, J.; Cardellach, M.E.; Rodríguez, H. Information content in reflected signals during GPS Radio Occultation observations Atmos. Meas. Tech. 2018, 11, 1883-1900. [CrossRef]

25. Sokolovskiy, S. Modeling and inverting radio occultation signals in the moist troposphere. Radio Sci. 2001, 36, 441-458. [CrossRef]

26. Beyerle G.; Hocke, K.; Wickert, J.; Schmidt, T.; Marquardt, C.; Reigber, C. GPS radio occultations with CHAMP: A radio holographic analysis of GPS signal propagation in the troposphere and surface reflections. J. Geophys. Res. 2002, 107, ACL-27. [CrossRef]

27. Sievert, T.; Rasch, J.; Carlström, A.; Pettersson, M.I. Analysis of reflections in GNSS radio occultation measurements using the phase matching amplitude. Atmos. Meas. Tech. 2018, 11, 569-580. [CrossRef]

28. Sokolovskiy, S.; Schreiner, W.; Zeng, Z.; Hunt, D.; Lin, Y.-C.; Kuo, Y.-H. Observation, analysis, and modeling of deep radio occultation signals, Effects of tropospheric ducts and interfering signals. Radio Sci. 2014, 49, 954-970. [CrossRef]

29. Healy, S.B.; Thepaut, J.-N. Assimilation experiments with CHAMP GPS radio occultation measurements. Q. J. R. Meteorol. Soc. 2006, 132, 605-623. [CrossRef]

30. Gorbunov, M.E.; Cardellach, E.; Lauritsen, K.B. Reflected ray retrieval from radio occultation data using radio holographic filtering of wave fields in ray space. Atmos. Meas. Tech. 2018, 11, 1181-1191. [CrossRef]

31. Wood, R.; Mechoso, C.R.; Bretherton, C.S.; Weller, R.A.; Huebert, B.; Straneo, F.; Albrecht, B.A.; Coe, H.; Allen, G.; Vaughan, G.; et al. The VAMOS Ocean-Cloud-Atmosphere-Land Study Regional Experiment (VOCALS-REx): Goals, platforms, and field operations. Atmos. Chem. Phys. 2011, 11, 627-654. [CrossRef]

32. Lopez, P. A 5-yr 40-km-resolution global climatology of superrefraction for ground-based weather radars. J. Appl. Meteorol. Climatol. 2009, 48, 89-110. [CrossRef]

33. Xie, F.; Wu, D.L.; Ao, C.O.; Kursinski, E.R.; Mannucci, A.J.; Syndergaard, S. Super-refraction effects on GPS radio occultation refractivity in marine boundary layers. Geophys. Res. Lett. 2010, 37, L11805. [CrossRef]

34. ROM SAF: ROM SAF Reflection Flag Database. 2016. Available online: http://www.romsaf.org/pub/ demo/reflection_flag/ (accessed on 20 February 2018).

35. Gorbunov, M.E.; Lauritsen, K.B.; Rhodin, A.; Tomassini, M.; Kornblueh, L. Radio holographic filtering, error estimation, and quality control of radio occultation data. J. Geophys. Res. 2006, 111, D10105. [CrossRef] 
36. Beckmann, P.; Spizzichino, A. The Scattering of Electromagnetic Waves from Rough Surface; Pergamon Press: Oxford, UK, 1963.

37. Stewart, R.H. Ocean-Wave Spectra. In Introduction To Physical Oceanography; Department of Oceanography, Texas A\&M University: College Station, TX, USA, 2008; pp. 284-288.

38. Jensen, A.S.; Lohmann, M.S.; Nielsen, A.S.; Benzon, H.-H. Geometrical optics phase matching of radio occultation signals. Radio Sci. 2004, 39, RS3009. [CrossRef]

(C) 2020 by the authors. Licensee MDPI, Basel, Switzerland. This article is an open access article distributed under the terms and conditions of the Creative Commons Attribution (CC BY) license (http://creativecommons.org/licenses/by/4.0/). 